\title{
Normal Product States for Fermions and Twisted Duality for CCR- and CAR-Type Algebras with Application to the Yukawa 2 Quantum Field Model
}

\author{
Stephen J. Summers \\ Fachbereich 4, Universität Osnabrück, D-4500 Osnabrück, \\ Federal Republic of Germany
}

\begin{abstract}
We present sufficient conditions that imply duality for the algebras of local observables in all Abelian sectors of all locally normal, irreducible representations of a field algebra if twisted duality obtains in one of these representations. It is verified that the Yukawa ${ }_{2}$ model satisfies these conditions, yielding the first proof of duality for the observable algebra in all coherent charge sectors in this model. This paper also constitutes the first verification of the assumptions of the axiomatic study of the structure of superselection sectors by Doplicher, Haag and Roberts in an interacting model with nontrivial sectors. The existence of normal product states for the free Fermi field algebra and, thus, the verification of the "funnel property" for the associated net of local algebras are demonstrated.
\end{abstract}

\section{Introduction}

In the algebraic approach to relativistic quantum field theory (for an introduction and motivation, see [24]), an intriguing property called duality became a natural object of study. For algebraic quantum field theory the basic structure is a net of *algebras $\{\mathfrak{U}(\mathcal{O})\}$ (generally taken to be von Neumann algebras, which we henceforth assume), wherein to each bounded space-time region $\mathcal{O}$ is associated an algebra $\mathfrak{A}(\mathcal{O})$. This net of algebras is required to satisfy certain properties that are physically motivated when one assumes that the self-adjoint elements of $\mathfrak{Q}(\mathcal{O})$ represent measurements performed in $\mathcal{O}$ (observables in $\mathcal{O}$ ). A crucial property is that of locality, which is an expression in this framework of the requirement that measurements in spacelike separated regions can not influence each other. Locality is represented mathematically by demanding that observables $A_{1}$ and $A_{2}$ localized in such spacelike separated regions $\mathcal{O}_{1}$ and $\mathcal{O}_{2}$ must commute, i.e. $\mathfrak{Q}\left(\mathcal{O}_{1}\right) \subset \mathfrak{H}\left(\mathcal{O}_{2}^{\prime}\right)^{\prime}$, where primes on algebras signify the commutant of the algebra and primes on spacetime regions signify the spacelike complement of the region. Duality strengthens this requirement by demanding that any observable that commutes with all observables 
localized in $\mathcal{O}^{\prime}$ must itself be associated with $\mathcal{O}$, i.e. $\mathfrak{U}(\mathcal{O})=\mathfrak{A}\left(\mathcal{O}^{\prime}\right)^{\prime}$. If duality is satisfied, the local algebras are maximal in the sense that if $\left\{\mathfrak{U}_{1}(\mathcal{O})\right\}$ and $\left\{\mathfrak{U}_{2}(\mathcal{O})\right\}$ are two nets of local algebras such that duality obtains for the system $\left\{\mathfrak{U}_{2}(\mathcal{O})\right\}$ and for every $\mathcal{O}, \mathfrak{U}_{1}(\mathcal{O}) \supseteq \mathfrak{U}_{2}(\mathcal{O})$, then $\left\{\mathfrak{U}_{1}(\mathcal{O})\right\}=\left\{\mathfrak{U}_{2}(\mathcal{O})\right\}$. This follows at once from the inclusions

$$
\mathfrak{U}_{2}(\mathcal{O}) \subseteq \mathfrak{U}_{1}(\mathcal{O}) \subseteq \mathfrak{U}_{1}\left(\mathcal{O}^{\prime}\right)^{\prime} \subseteq \mathfrak{U}_{2}\left(\mathcal{O}^{\prime}\right)^{\prime}
$$

Duality has been employed in the axiomatic study by Doplicher, Haag and Roberts (DHR) $[12,13]$ of the structure of superselection sectors for compact gauge groups of the first kind. It was shown in [35] that it suffices for the application in [12, 13] that the weaker condition of essential duality, requiring that the algebras $\mathfrak{B}(\mathcal{O})=\mathfrak{A}\left(\mathcal{O}^{\prime}\right)^{\prime}$ satisfy duality, obtains. (This then necessitates an extension of the representations of the original net of algebras - see [35].) In [6,7] sufficient conditions (that are probably satisfied in all reasonable quantum field models) were given that assure that duality holds for the canonical algebras (the algebras generated by bounded functions of the fields with support in the appropriate region) associated with spacelike wedges, but only essential duality could be shown for algebras associated with double cones. Although it is thus under generally valid conditions possible to find $a$ net of algebras with which one can do the DHR theory, the question of what is the appropriate net of observable algebras is begged. If, however, the "canonical" net, which is one of the smallest candidates (at least if one is dealing with quantum field models), satisfies duality, it equals the BisognanoWichmann net and there is no local extension.

As it has been found useful in physics to introduce unobservable fields (chargecarrying fields, fermions) whose locality is not expressed through commutation relations, one has defined a transformation $\mathfrak{F}(\mathcal{O}) \rightarrow \mathfrak{F}^{t}(\mathcal{O})$, a "twist" related to a Klein transformation, of the local field algebra (von Neumann algebra generated by the observable and unobservable fields with support in $\mathcal{O})$ so that one has the relation $\mathfrak{F}(\mathcal{O}) \subset \mathfrak{F}^{t}\left(\mathcal{O}^{\prime}\right)^{\prime}$-called twisted locality. The requirement of equality, called twisted duality, is closely related to duality for the observable algebras (see $[12,13]$ and Chapter II.

Araki's original proof of duality for the free Bose field [1] was subsequently simplified and refined in a series of papers $[29,15,25]$ and extended to free fields of mass $m>0$ and any integer spin by Dell'Antonio [11]. Using Dell'Antonio's results on the structure of Fermi systems, twisted duality for free Fermi fields was proven in [12] (see also [16]). Duality for algebras associated with certain space-time regions was proven for free, massless, integer spin fields in [25]. Although all these proofs were carried out for four space-time dimensions, all proofs for the massive theories can be reproduced in two space-time dimensions.

In [14] Driessler demonstrated duality for the first time in an interacting quantum field model $-P(\phi)_{2}$ - by isolating sufficient conditions that assure that the knowledge of duality in one locally normal representation of CCR-algebra can be carried over to all other locally normal representations of the CCR-algebra and then verifying that the Fock space representation of the relativistic Bose field fulfills these conditions, thus yielding duality for $P(\phi)_{2}$ models due to their local Fock property (which implies they are locally normal representations of the CCR-algebra). 
However, as he shows, his conditions exclude the existence of nontrivial locally generated superselection sectors. In this paper we propose to generalize Driessler's work to examine field algebras and twisted duality and to apply the result to models with fermions and nontrivial superselection sectors. In particular we shall apply our theorem to the scalar (or pseudoscalar) Yukawa ${ }_{2}\left(+P\left(\phi_{2}\right)\right.$ quantum field model, which is also known to have the local Fock property, enabling us to use the wellstudied Fock representation for the reference representation.

More specifically, we find sufficient conditions on a net of local field algebras $\{\mathfrak{F}(\mathcal{O})\}$ (i.e. admitting nonobservable fields) generating a $C^{*}$-algebra $\mathscr{F}$, so that if twisted duality (and one technical condition) is satisfied in one locally normal representation of this algebra, duality will be satisfied by the observable algebras on all Abelian sectors (see [12]) of all locally normal representations of the said algebra. Twisted duality itself will be recovered in the latter representations whenever the observable algebra is obtained from the field algebra as the fixed points under gauge transformations of an Abelian gauge group, i.e. the observables are gauge invariant under this Abelian group. (We also present a slightly different version of the technical condition that assures twisted duality for a nonabelian gauge group.) In particular, we shall require that $\{\mathfrak{F}(\mathcal{O})\}$ satisfies the funnel property. This property, a variant of a conjecture of Borchers for all relativistic quantum field theories, asserts that for an ascending sequence $\left\{\mathcal{O}_{n}\right\} \uparrow \mathbb{R}^{d}$ of space-time regions there exists a sequence of type I factors $\left\{\mathfrak{M}_{n}\right\}$ such that

$$
\mathfrak{F}\left(\mathcal{O}_{n}\right) \subset \mathfrak{M}_{n} \subset \mathfrak{F}\left(\mathcal{O}_{n+1}\right)
$$

for every $n$. We remark that in a free relativistic quantum field theory, the $\mathfrak{F}(\mathcal{O})$ are typically type III factors $[2,11]$. The abstract theorem is stated and proven in Chap. II, and we refer the reader there for further details.

We wish then to apply this theorem to concrete relativistic quantum field models. In this application, the local field algebras will be the canonical algebras associated to local quantum fields, and we shall take as our reference representation the well-studied Fock (free) representation of the relativistic Bose and Fermi fields (which verifies twisted duality and the technical assumption imposed on the reference representation-see Chap. IV). The funnel property was demonstrated for the local algebras associated with the free Bose field by Buchholz [8] (when one takes into account the validity of duality), and we do the same here for the free Fermi field algebras, capitalizing on ideas employed by him. Buchholz showed that under the standard assumptions of local relativistic quantum theory, the existence of normal product states yields a form of the funnel property. That is to say, if for a suitable pair of spacelike separated regions $\mathcal{O}_{1}, \mathcal{O}_{2}$, and a pair of normal states $\phi_{1}$ of $\mathfrak{U}\left(\mathcal{O}_{1}\right)$ and $\phi_{2}$ of $\mathfrak{U}\left(\mathcal{O}_{2}\right)$, there exists a normal state $\phi$ on $\mathfrak{U}$ such that $\phi\left(\sum A_{n} B_{n}\right)=$ $\Sigma \phi_{1}\left(A_{n}\right) \phi_{2}\left(B_{n}\right)$ for all $A_{n} \in \mathfrak{U}\left(\mathcal{O}_{1}\right)$ and $B_{n} \in \mathfrak{A}\left(\mathcal{O}_{2}\right)$, then there exist type I algebras $\mathfrak{M}_{1}, \mathfrak{M}_{2}$, such that $\mathfrak{A}\left(\mathcal{O}_{1}\right) \subset \mathfrak{M}_{1} \subset \mathfrak{M}_{2}^{\prime} \subset \mathfrak{A}\left(\mathcal{O}_{2}\right)^{\prime}$ (which is actually the form of Borchers' conjecture). Duality then yields the funnel property. In the presence of fermions, this result is again valid if $\mathfrak{A}\left(\mathcal{O}_{1}\right)$ is replaced by $\mathfrak{F}\left(\mathcal{O}_{1}\right)$ and $\mathfrak{A}\left(\mathcal{O}_{2}\right)$ by $\mathfrak{F}^{t}\left(\mathcal{O}_{2}\right)$. Twisted duality then entails the funnel property. The existence of normal product states is verified for the free Fermi algebra in Chap. III.

In Chap. IV the remaining assumptions of the abstract theorem proven in 
Chap. II are demonstrated for the $Y_{2}+P(\phi)_{2}$ model, using results proven in the Hamiltonian formalism (see [19, 21, 39, 40, 17]) and the Euclidean formalism (see $[42,43,10,26,49])$. Unfortunately, we will need properties proven in one or the other formalism, but not in both, so we shall be obliged to prove the equivalence of the resulting models. We verify the necessary properties in the weak coupling limit, but the same ideas can be applied to the strong coupling limit of Balaban and Gawedzki [4], as we shall point out in a bit more detail in Chap. IV.

\section{The Funnel Property and Twisted Duality}

In this chapter we shall generalize a theorem of Driessler [14] to determine sufficient conditions on a given irreducible, locally normal representation of a field algebra $\mathscr{F}$, here admitting unobservable (Fermi) fields, that permit one to conclude from the knowledge that twisted duality obtains in this representation that duality holds for the observable algebra in all Abelian coherent sectors of all irreducible, locally normal representations of $\mathscr{F}$, i.e. duality holds for all irreducible, locally normal representations of the observable algebra $\mathscr{A}$ (given by the fixed points of $\mathscr{F}$ under a gauge group $\mathscr{G}$ ) that admit one-dimensional unitary representations of $\mathscr{G}$. If the group $\mathscr{G}$ is Abelian, twisted duality itself will be regained in all the mentioned representations of $\mathscr{F}$. Slightly stronger conditions will be given that yield twisted duality for any compact gauge group (see Theorem 2.8). These results are an advance with respect to [14] because this theorem may be applied to relativistic quantum theories with non-trivial superselection sectors and fermions. The general setting of this study is the work of Doplicher, Haag and Roberts $[12,13]$, and we refer the reader there for further details and motivations, if necessary.

To begin, we introduce the mathematical objects that shall concern us. We assume a correspondence $\mathcal{O} \rightarrow \mathfrak{F}(\mathcal{O})$ between arbitrary bounded open sets $\mathcal{O} \subset \mathbb{R}^{d}(\mathrm{a}$ special subclass of such regions - the bounded double cones (bdc's) - shall often be singled out) and $W^{*}$-algebras $\mathfrak{F}(\mathcal{O})$, embedded in a $C^{*}$-algebra $\mathscr{F}$ that is the norm closure of the inductive limit $\bigcup_{\mathcal{O} \subset \mathbb{B}^{d}} \mathfrak{F}(\mathcal{O})$, such that isotony obtains $\left(\mathcal{O}_{1} \subset \mathcal{O}_{2} \Rightarrow \mathfrak{F}\left(\mathcal{O}_{1}\right) \subset \mathfrak{F}\left(\mathcal{O}_{2}\right)\right)$. $\mathscr{F}\left(\mathcal{O}^{\prime}\right)$ will denote the $C^{*}$-subalgebra of $\mathscr{F}$ generated by

$\bigcup_{\mathcal{O}_{0} \subset \mathcal{O}^{\prime}} \mathfrak{F}\left(\mathcal{O}_{0}\right)\left(\mathcal{O}^{\prime}\right.$ signifies the interior of the causal complement of $\left.\mathcal{O}\right)$. One assumes further a faithful representation $g \rightarrow \alpha_{g}$ of a compact group $\mathscr{G}$ by automorphisms of $\mathscr{F}$ that act strictly locally:

$$
\alpha_{g}(\mathfrak{F}(\mathcal{O}))=\mathfrak{F}(\mathcal{O}), \quad \forall \mathcal{O}
$$

We presume the map $g \rightarrow \alpha_{g}(F)$ to be ultraweakly continuous for $F \in \mathfrak{F}(\mathcal{O})$, that is to say, then, locally ultraweakly continuous (i.e. continuous in the weak topology induced by the locally normal linear forms). The observables are defined to be the gauge invariant elements of $\mathscr{F}$ :

$$
\mathfrak{A}(\mathcal{O})=\left\{F \in \mathfrak{F}(\mathcal{O}) \mid \alpha_{g}(F)=F, \quad \forall g \in \mathscr{G}\right\},
$$

with $\mathscr{A}$ and $\mathscr{A}\left(\mathcal{O}^{\prime}\right)$ given analogously. The closure properties of the algebras are clearly maintained. 
The unobservable fields do not in general express their local nature through commutation relations, but through anticommutation relations. We thus presume the following local structure. The field algebra $\mathscr{F}$ is assumed to have Bose-Fermi commutation relations, i.e. we suppose the existence of a $g_{0} \in \mathscr{G}$ with $g_{0}^{2}=e$ so that, setting $F_{+}=\frac{1}{2}\left(F+\alpha_{g_{0}}(F)\right)$ and $F_{-}=\frac{1}{2}\left(F-\alpha_{g_{0}}(F)\right)$,

$$
\begin{aligned}
& F_{+} F_{+}^{\prime}-F_{+}^{\prime} F_{+}=0 \\
& F_{+} F_{-}^{\prime}-F_{-}^{\prime} F_{+}=0, \quad F \in \mathfrak{F}\left(\mathcal{O}_{1}\right), \quad F^{\prime} \in \mathfrak{F}\left(\mathcal{O}_{2}\right), \quad \mathcal{O}_{1} \subset \mathcal{O}_{2}^{\prime} . \\
& F_{-} F_{-}^{\prime}+F_{-}^{\prime} F_{-}=0
\end{aligned}
$$

In the following we shall be interested in irreducible, locally normal representations $\pi$ of $\mathscr{F}$ as a concrete $C^{*}$-algebra $\pi(\mathscr{F})$ on a Hilbert space $\mathscr{H}_{\pi}$. And because duality fails in the presence of spontaneous symmetry breaking [34], it is no loss of generality for our purposes (given the continuity assumption on $\alpha_{g}$ above) to assume the existence of a faithful strongly continuous unitary representation $g \rightarrow$ $U_{\pi}(g) \in \mathfrak{B}\left(\mathscr{H}_{\pi}\right)$ (the bounded operators on $\mathscr{H}_{\pi}$ ) implementing the group of automorphisms $\alpha_{g}$ (although for the purposes of formulating and proving twisted duality, it suffices that at least the automorphism $\alpha_{g_{0}}$ be unitarily implemented):

$$
\pi\left(\alpha_{g}(F)\right)=U_{\pi}(g) \pi(F) U_{\pi}(g)^{*}, \quad F \in \mathscr{F}, \quad g \in \mathscr{G} .
$$

A representation with such a unitary implementation of the gauge group shall be called a gauge representation. As a form of shorthand, we rewrite the information in (2.3) as follows. With $g_{0}$ the particular element of $\mathscr{G}$ that was singled out in (2.3), we define $\left(\mathfrak{F}_{\pi}(\mathcal{O}) \equiv \pi(\mathfrak{F}(\mathcal{O}))\right)$

$$
\mathbb{F}_{\pi}^{t}(\mathcal{O})=Z_{\pi} \mathfrak{F}_{\pi}(\mathcal{O}) Z_{\pi}^{-1}
$$

with

$$
Z_{\pi}=\frac{1-i}{2}+\frac{1+i}{2} U_{\pi}\left(g_{0}\right)
$$

a unitary operator. With this definition, (2.3) implies that

$$
\mathfrak{F}_{\pi}\left(\mathcal{O}_{1}\right) \subset \mathfrak{F}_{\pi}^{t}\left(\mathcal{O}_{2}\right)^{\prime}, \quad \mathcal{O}_{1} \subset \mathcal{O}_{2}^{\prime},
$$

which is called "twisted locality". We note that $\mathfrak{F}_{\pi}^{t}(\mathcal{O})$ is spatially isomorphic to $\mathfrak{F}_{\pi}(\mathcal{O})$ (we write $\mathscr{F}_{\pi}^{t}(\mathcal{O})=t_{\pi}(\mathscr{F}(\mathcal{O}))$ ). $\mathscr{F}_{\pi}^{t}\left(\mathcal{U}^{\prime}\right)$ and $\mathscr{F}_{\pi}^{t}$ are defined analogously to $\mathscr{F}_{\pi}\left(\mathcal{O}^{\prime}\right)$ and $\mathscr{F}_{\pi}$ and are clearly spatially isomorphic to their counterparts.

The funnel property that is crucial in the proof to follow is defined explicitly as follows. (We signify with $\mathcal{O}_{1} \ll \mathcal{O}_{2}$ the relation $\overline{\mathcal{O}}_{1} \subset \mathcal{O}_{2}$, where $\overline{\mathcal{O}}$ denotes the closure of $\mathscr{O}$ and $\mathcal{O}_{1}$ and $\mathcal{O}_{2}$ are required to have bases at a common time.)

Definition. $\mathscr{F}$ is said to have the funnel property if for all pairs of bounded double cones (bdc's) $\mathfrak{O}_{1}, \mathscr{O}_{2}$ with $\mathscr{O}_{1} \ll \mathscr{O}_{2}$ there exists a type I factor $\mathfrak{M}$ such that $\mathfrak{F}\left(\mathbb{O}_{1}\right) \subset$ $\mathfrak{M} \subset \mathfrak{F}\left(\mathcal{O}_{2}\right)$.

One notes at once that because factors contain no proper ultraweakly closed two-sided ideals and because normal representations are ultraweakly continuous, it follows that the restriction of every locally normal homomorphism to a local algebra $\mathfrak{F}\left(\mathcal{O}_{1}\right)\left(\subset \mathfrak{M} \subset \mathfrak{F}\left(\mathcal{O}_{2}\right)\right.$ for some type I factor $\left.\mathfrak{M}\right)$ is an isomorphism, so that within the class of representations we consider, the local von Neumann algebras 
$\mathfrak{F}(\mathcal{O})$ and $\mathfrak{M}$ are representation independent, i.e., $\pi_{1}(\mathfrak{F}(\mathcal{O})) \simeq \pi_{2}(\mathfrak{F}(\mathcal{O}))\left(\pi_{1}(\mathfrak{M} \simeq\right.$ $\pi_{2}(\mathfrak{M})$ ) for any two locally normal representations $\pi_{1}, \pi_{2}$ of $\mathscr{F}$. Furthermore, since for any ascending sequence of bdc's $\left\{\mathcal{O}_{m}\right\} \uparrow \mathbb{R}^{d}$ (with $\left.\mathcal{O}_{m} \ll \mathcal{O}_{m+1}\right), \cup\left\{\mathfrak{F}\left(\mathcal{O}_{m}\right)\right\}$ is uniformly dense in $\mathscr{F}$, there exists an associated ascending sequence of type I factors $\left\{\mathfrak{M}_{m}\right\}$ such that $\cup\left\{\mathfrak{M}_{m}\right\}$ is dense in $\mathscr{F}$. But, since $\pi_{1}\left(\mathfrak{M}_{m}\right) \simeq \pi_{2}\left(\mathfrak{M}_{m}\right)$ for any $m$ and any locally normal representations $\pi_{1}, \pi_{2}$ of $\mathscr{F}$, Prop. 1.23.4 in [38] informs us that $\pi_{1}(\mathscr{F})$ and $\pi_{2}(\mathscr{F})$ are isomorphic as $C^{*}$-algebras. These assertions are all valid for $\mathscr{F}^{t}$ as well, since $t\left(\mathfrak{M}_{m}\right)$ is a type I factor. Thus, the algebras $\mathscr{F}$ and $\mathscr{F}^{t}$ (and thus $\mathscr{F}\left(\mathcal{O}^{\prime}\right)$, $\mathscr{F}^{t}\left(\mathcal{O}^{\prime}\right)$ ) are independent of representation (for the representations that we consider), and we drop the subscripts $\pi$ in future. However, in (2.6) and in the condition for twisted duality, $\mathfrak{F}(\mathcal{O})=\mathscr{F F}^{t}\left(\mathcal{O}^{\prime}\right)_{\pi}^{\prime}$, the commutant is representation-dependent, and we shall be careful to indicate the representation wherever necessary (we write henceforth $\mathfrak{F}(\mathcal{O})_{\pi}^{\prime}$ instead of $\mathfrak{F}_{\pi}(\mathcal{O})^{\prime}$ to emphasize that the representation dependence comes from the taking of commutants).

We point out, for later use, that the algebras $\mathscr{F}$ and $\mathscr{F}^{t}$ can be realized naturally as isomorphic subalgebras of the covariance algebra $\mathscr{F} \otimes_{\alpha} \mathscr{G}_{0}$, which is the crossed product [50] of $\mathscr{F}$ with the subgroup $\mathscr{G}_{0} \subset \mathscr{G}$ consisting of the elements $\left\{e, g_{0}\right\}$. $\mathscr{F} \otimes_{\alpha} \mathscr{G}_{0}$ is a uniquely determined $C^{*}$-algebra, and its representations $\left(\mathscr{H}_{\rho}, \rho\right)$ are in $1-1$ correspondence with the covariant representations $\left(\mathscr{H}_{\pi}, \pi, U_{\pi}\right)$ of the triple $\left(\mathscr{F}, \mathscr{G}_{0}, \alpha\right)$. Furthermore, one knows that $\rho\left(\mathscr{F} \otimes_{\alpha} \mathscr{G}_{0}\right)_{\mathscr{H}_{\rho}}^{\prime \prime}=\{\pi(\mathscr{F})$, $\left.U_{\pi}\left(\mathscr{G}_{0}\right)\right\}_{\mathscr{H}_{\pi}}^{\prime \prime}\left(\mathscr{H}_{\rho} \simeq \mathscr{H}_{\pi}\right)$ for every such pair.

If $F \in \mathfrak{B}\left(\mathscr{H}_{\pi}^{\rho}\right)$, define $m_{\pi}(F)=\int_{\mathscr{G}} U_{\pi}(g) F U_{\pi}(g)^{-1} d \mu(g)$, where $\mu$ denotes the normalized Haar measure on $\mathscr{G}$ and the integral is understood in the weak operator topology. Then one knows [12] that $m_{\pi}$ defines a normal positive linear mapping of $\mathfrak{B}\left(\mathscr{H}_{\pi}\right)$ onto $U_{\pi}(\mathscr{G})^{\prime}$ satisfying $m_{\pi}(\mathscr{L})=\mathscr{L} \cap U_{\pi}(\mathscr{G})^{\prime}$ for every ${ }^{*}$-algebra $\mathscr{L}$ on $\mathscr{H}_{\pi}$ such that $m_{\pi}(\mathscr{L}) \subset \mathscr{L}$; therefore, using the normality of $m_{\pi}, m_{\pi}(\mathscr{L})^{\prime \prime}=m_{\pi}\left(\mathscr{L}^{\prime \prime}\right)=$ $\mathscr{L}^{\prime \prime} \cap U_{\pi}(\mathscr{G})^{\prime}$. Thus one has

$$
\begin{aligned}
& \mathfrak{U}(\mathcal{O})=m_{\pi}(\mathfrak{F}(\mathcal{O}))=m_{\pi}\left(\mathfrak{F}^{t}(\mathcal{O})\right), \\
& \mathscr{A}\left(\mathcal{O}^{\prime}\right)=m_{\pi}\left(\mathscr{F}\left(\mathcal{O}^{\prime}\right)\right)=m_{\pi}\left(\mathscr{F}^{t}\left(\mathcal{O}^{\prime}\right)\right) \\
& \mathscr{A}\left(\mathcal{O}^{\prime}\right)_{\pi}^{\prime \prime}=m_{\pi}\left(\mathscr{F}\left(\mathcal{O}^{\prime}\right)_{\pi}^{\prime \prime}\right)=m_{\pi}\left(\mathscr{F}^{t}\left(\mathcal{O}^{\prime}\right)_{\pi}^{\prime \prime}\right) \text {. } \\
& \mathscr{A}=m_{\pi}(\mathscr{F}) \quad=m_{\pi}\left(\mathscr{F}^{t}\right), \\
& \mathscr{A}^{\prime \prime}=m_{\pi}\left(\mathscr{F}_{\pi}^{\prime \prime}\right) \quad=m_{\pi}\left(\mathscr{F}_{\pi}^{t \prime \prime}\right) \text {. }
\end{aligned}
$$

One of the two main theorems of this chapter is the following.

Theorem 2.1. Let $\mathscr{F}$ have the funnel property and assume, in addition:

(1) There exists an irreducible, locally normal, gauge representation (ilngr) $\pi_{0}$ of $\left(\mathscr{F}, \mathscr{G}, \alpha, \mathscr{F}^{t}\right)$ in which twisted duality holds for all bdc's, i.e.

$$
\mathscr{F}\left(\mathcal{O}^{\prime}\right)_{\pi_{0}}^{\prime \prime}=\mathscr{F}^{t}(\mathcal{O})_{\pi_{0}}^{\prime}, \quad \forall \mathcal{O b d c}
$$

(2) For any bdc's $\mathcal{O}, \mathcal{O}_{1}$ such that $\mathcal{O} \ll \mathcal{O}_{1}$ there exists a bdc $\mathcal{O}_{2}$ with $\mathcal{O}_{1} \ll \mathcal{O}_{2}$ such that

$$
\pi_{0}\left(\mathfrak{F}^{t}(\mathcal{O})^{c} \cap \mathfrak{F}\left(\mathcal{O}_{1}\right)\right) \subset\left[\mathscr{F}\left(\mathcal{O}^{\prime}\right) \cap \mathfrak{F}\left(\mathcal{O}_{2}\right)\right]_{\pi_{0}}^{\prime \prime}
$$


where $\mathfrak{F}^{t}(\mathcal{O})^{c}=\mathfrak{F}^{t}(\mathcal{O})^{\prime} \cap \mathscr{F}^{t}$ is a representation-independent $C^{*}$-algebra. Then

$$
\mathscr{A}\left(\mathcal{O}^{\prime}\right)_{\pi}^{\prime \prime}=m_{\pi}\left\{\mathfrak{F}(\mathcal{O})_{\pi}^{\prime}\right\}, \quad \forall \mathcal{O b d c}
$$

for every ilngr $\pi$ of $\left(\mathscr{F}, \mathscr{G}, \alpha, \mathscr{F}^{t}\right)$.

Remarks. (1) The condition (2) in the special case of the absence of fermions is stronger than condition (3) in Driessler's Theorem 1 [14], but we may do without the counterpart of his condition (2) and simplify a portion of the proof. We expect, in any case, that wherever this theorem can in practice be applied, condition (2) above is sufficiently general.

(2) Note that the requirement in [14] that $\mathscr{A}$ be simple is not necessary.

(3) We do not know at present how to prove a similar result when both $\mathcal{O}$ and $\mathcal{O}^{\prime}$ are unbounded, for example, if $\mathcal{O}$ and $\mathcal{O}^{\prime}$ are the right and left wedges of $[6,7]$.

Before we prove this theorem, we recall a result from [33] that clarifies the significance of the conclusion of Theorem 2.1.

Theorem 2.2. Let $\mathcal{O}$ be a bdc. Then the following are equivalent:

a) $m_{\pi}\left(\mathscr{F}\left(\mathcal{O}^{\prime}\right)_{\pi}^{\prime}\right)=\mathfrak{U}(\mathcal{O})_{\pi}$.

b) $m_{\pi}\left(\mathfrak{F}(\mathcal{O})_{\pi}^{\prime}\right)=\mathscr{A}\left(\mathcal{O}^{\prime}\right)_{\pi}^{\prime \prime}$.

c) Duality holds for $\mathcal{O}$ in the vacuum (charge 0 ) sector in $\mathscr{H}_{\pi}$.

d) Duality holds for $\mathcal{O}$ in every Abelian sector in $\mathscr{H}_{\pi}$.

Remarks. (1) One calls a sector (a subspace of $\mathscr{H}_{\pi}$ reducing $\mathscr{A}$ irreducibly) Abelian if it corresponds to a one-dimensional representation of $\mathscr{G}$ (see [12]).

(2) We have defined the algebras $\mathscr{F}\left(\mathcal{O}^{\prime}\right)$ using the directed set of bounded open sets in $\mathbb{R}^{2}$, avoiding a problem in the proof of this theorem noticed in [36].

Thus, although we are not able to conclude twisted duality in the ilngr $\pi$, we do obtain the desired duality of the observable algebras wherever it is possible. Indeed, recalling Theorem 5.6 from [12], duality will not obtain in non-Abelian sectors. For an Abelian gauge group $\mathscr{G}$ one has, as remarked by Roberts [33]:

Theorem 2.3. If $\mathscr{G}$ is Abelian and $m_{\pi}\left(\mathscr{F}\left(\mathcal{O}^{\prime}\right)_{\pi}^{\prime}\right)=\mathfrak{U}(\mathcal{O})_{\pi}$, then $\pi\left(\mathscr{F}\left(\mathcal{O}^{\prime}\right)\right)^{\prime \prime}=\mathscr{F}^{t}(\mathcal{O})_{\pi}^{\prime}$.

Proof. Implicit in the proof of Proposition 6.2 of [13].

Thus, twisted duality is regained for Abelian gauge groups. See, however, Theorem 2.8 .

Before we undertake the proof of Theorem 2.1, we need to introduce some additional definitions. Let $\mathcal{O}$ be the bdc for which we wish to prove duality and choose a sequence $\left\{\mathcal{O}_{n}\right\}$ of bdc's such that $\bigcap_{n} \mathcal{O}_{n}=\overline{\mathcal{O}}$ and $\mathcal{O}_{n+1} \ll \mathcal{O}_{n}$. The funnel property gives us a sequence $\left\{\mathfrak{M}_{n}\right\}$ of type I factors so that $\mathfrak{F}\left(\mathcal{O}_{n}\right) \supset \mathfrak{M}_{n} \supset \mathfrak{F}\left(\mathcal{O}_{n+1}\right)$. The sequence $\left\{\mathfrak{M}_{n}\right\}$ is decreasing and $\bigcap_{n} \mathfrak{M}_{n}=\mathfrak{F}(\mathcal{O}), \bigcap_{n} \mathfrak{M}_{n}^{t}=\mathfrak{F}^{t}(\mathcal{O})$, which can be readily verified using the twisted duality in $\pi_{0}$. We choose another sequence of bdc's 
$\left\{\tilde{\mathcal{O}}_{k}\right\} \uparrow \mathbb{R}^{d}$ so that $\tilde{\mathcal{O}}_{k} \ll \tilde{\mathcal{O}}_{k+1}$ and $\tilde{\mathcal{O}}_{k} \gg \mathcal{O}_{n}$ for all $k, n$. Thus, there exists a sequence $\left\{\mathfrak{N}_{k}\right\}$ of type I factors with $\mathfrak{F}\left(\widetilde{\mathcal{O}}_{k}\right) \subset \mathfrak{N}_{k} \subset \mathfrak{F}\left(\tilde{\mathcal{O}}_{k+1}\right)$ (similarly for $\left.\left\{\mathfrak{N}_{k}^{t}\right\}\right)$. Thus, $\mathscr{F}=$ $\overline{\cup \mathfrak{N}}_{k}^{n}$ and $\mathscr{F}^{t}=\overline{\cup \mathfrak{N}}_{k}^{t n}$, where the superscript $-n$ signifies closure in the norm topology.

Lemma 2.4. Let $\pi$ be an ilngr of $\left(\mathscr{F}, \mathscr{G}, \alpha, \mathscr{F}^{t}\right)$. Then for any $\mathfrak{M}_{n}$ we have $\left(\mathfrak{M}_{n}^{c}\right)_{\pi}^{\prime \prime}=$ $\left(\mathfrak{M}_{n}\right)_{\pi}^{\prime}$ and $\left(\mathfrak{M}_{n}^{t c}\right)_{\pi}^{\prime \prime}=\left(\mathfrak{M}_{n}^{t}\right)_{\pi}^{\prime}$, where $\mathfrak{M}_{n}^{c}=\mathfrak{M}_{n}^{\prime} \cap \mathscr{F}$ and $\mathfrak{M}_{n}^{t c}=\left(\mathfrak{M}_{n}^{t}\right)^{\prime} \cap \mathscr{F}$.

Proof. The proof is the same as that of Lemma 1 in [14], but we feel some more details should be indicated for the reader's convenience. (We thank Prof. Driessler for a conversation about this proof.) We have $\mathfrak{M}_{n}^{c}=\mathscr{F} \cap \mathfrak{M}_{n}^{\prime} \supset \mathfrak{N}_{k} \cap \mathfrak{M}_{n}^{\prime}$, for every $k$. But $\mathfrak{N}_{k}$ a type I factor implies $\mathfrak{N}_{k} \simeq \mathfrak{B}\left(\mathscr{H}_{k}\right)$ for some Hilbert space $\mathscr{H}_{k}$. Defining $\mathfrak{N}_{k} \cap \mathfrak{M}_{n}^{\prime} \equiv \mathfrak{M}_{n}^{c(k)}$, one observes (since $\mathfrak{M}_{n} \subset \mathfrak{N}_{k}$ for every $\left.n, k\right)$ that $\left(\mathfrak{M}_{n} \cup \mathfrak{M}_{n}^{c(k)}\right)_{k}^{\prime \prime}=\mathfrak{N}_{k}$, since $\mathfrak{M}_{n}$ is a factor. Thus, $\left(\left(\mathfrak{M}_{n}^{c}\right)_{\pi}^{\prime \prime} \cup \mathfrak{M}_{n}\right)_{\pi}^{\prime \prime} \supset\left(\mathfrak{M}_{n} \cup \mathfrak{M}_{n}^{c(k)}\right)_{k}^{\prime \prime}=\mathfrak{N}_{k}$, for every k. Thus, $\left(\left(\mathfrak{M}_{n}^{c}\right)_{\pi}^{\prime \prime} \cup \mathfrak{M}_{n}\right)_{\pi}^{\prime \prime}=\mathfrak{B}\left(\mathscr{H}_{\pi}\right)$, which implies $\left(\mathfrak{M}_{n}^{c}\right)_{\pi}^{\prime \prime}=\left(\mathfrak{M}_{n}\right)_{\pi}^{\prime}$, since type I factors are coupled. The proof of the second assertion above is similar, noting that $\mathscr{F}$ weakly dense in $\mathfrak{B}\left(\mathscr{H}_{\pi}\right)$ implies $\mathscr{H}^{t}$ is weakly dense in $\mathfrak{B}\left(\mathscr{H}_{\pi}\right)$.

Lemma 2.5. In every ilngr $\pi$ of $\left(\mathscr{F}, \mathscr{G}, \alpha, \mathscr{F}^{t}\right)$ we have $\left[\pi\left(\mathfrak{F}(\mathcal{O})^{c}\right)\right]^{\prime \prime}=\mathfrak{F}(\mathcal{O})_{\pi}^{\prime}$ and $\left[\pi\left(\mathfrak{F}^{t}(\mathcal{O})^{c}\right)\right]^{\prime \prime}=\mathfrak{F}^{t}(\mathcal{O})_{\pi}^{\prime}$.

Proof. Same as Lemma 2 in [14].

Lemma 2.6. $\mathfrak{F}(\mathcal{O})^{c}=\overline{\cup\left(\mathfrak{F}(\mathcal{O})^{c} \cap \mathfrak{N}_{k}\right)^{n}}$ and $\mathfrak{F}^{t}(\mathcal{O})^{c}=\overline{\left.U\left(\mathfrak{F}^{t}(\mathcal{O})^{c} \cap \mathfrak{N}_{k}^{t}\right)\right)^{n}}$.

Proof. Same as Lemma 3 in [14].

Lemma 2.7. For every ilngr $\pi$ of $(\mathscr{F}, \mathscr{G}, \alpha, \mathscr{F} t)$, we have

$$
\left\{\bigcup_{k}\left[m_{\pi}\left\{\mathfrak{F}^{t}(\mathcal{O})^{c} \cap \mathfrak{F}\left(\tilde{\mathcal{O}}_{k}\right)\right\}\right]\right\}^{-n}=m_{\pi}\left\{\mathfrak{F}^{t}(\mathcal{O})^{c}\right\} .
$$

Proof. Because $\left\{\widetilde{F}^{t}(\mathcal{O})^{c} \cap \mathfrak{F}\left(\widetilde{\mathcal{O}}_{k}\right)\right\}$ forms an ascending sequence, the lefthand side above equals

$$
\begin{aligned}
& \operatorname{n-lim}_{k \rightarrow \infty} m_{\pi}\left\{\mathfrak{F}^{t}(\mathcal{O})^{c} \cap \mathfrak{F}\left(\tilde{\mathcal{O}}_{k}\right)\right\} \\
& =\underset{k \rightarrow \infty}{\mathrm{n}-\lim } m_{\pi}\left\{\mathfrak{F}^{t}(\mathcal{O})^{c} \cap \mathscr{A} \cap \mathfrak{F}\left(\tilde{\mathcal{O}}_{k}\right)\right\},
\end{aligned}
$$

since $\quad m_{\pi}\left\{\mathfrak{F}^{t}(\mathcal{O})^{c} \cap \mathfrak{F}\left(\widetilde{\mathcal{O}}_{k}\right)\right\} \subset \mathfrak{F}^{t}(\mathcal{O})^{c} \cap \mathfrak{F}_{(}\left(\widetilde{\mathcal{O}}_{k}\right), \quad U_{\pi}(\mathscr{G})^{\prime}=\mathscr{A}_{\pi}^{\prime \prime} \quad$ and $\quad \mathbb{F}^{t}(\mathcal{O})^{c} \cap \mathscr{A}_{\pi}^{\prime \prime}=$ $\mathbb{F}^{t}(\mathcal{O})^{c} \cap \mathscr{A}$. But $\mathscr{A} \cap \mathfrak{F}\left(\widetilde{\mathcal{O}}_{k}\right)=\mathscr{A} \cap \mathfrak{F}^{t}\left(\widetilde{\mathcal{O}}_{k}\right)$, so that $(2.8)$ equals

$$
\begin{aligned}
& \mathrm{n}_{k \rightarrow \infty} m_{\pi}\left\{\mathscr{F}^{t}(\mathcal{O})^{c} \cap \mathfrak{F}^{t}\left(\tilde{\mathcal{O}}_{k}\right)\right\}
\end{aligned}
$$

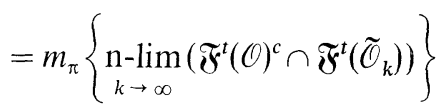

$$
\begin{aligned}
& =m_{\pi}\left\{\mathbb{F}^{t}(\mathcal{O})^{c}\right\} \text {, }
\end{aligned}
$$


which follows directly from Lemma 2.6 when one recalls that $\mathfrak{N}_{k-1}^{t} \subset \mathfrak{F}^{t}\left(\tilde{\mathcal{O}}_{k}\right) \subset$ $\mathfrak{N}_{k}^{t} \subset \mathfrak{F}^{t}\left(\tilde{\mathbb{O}}_{k+1}\right)$.

Proof of Theorem 2.1. We first assert that for every $k$, there is a bdc $\tilde{\mathcal{O}}_{\mathfrak{N}_{k}}$ so that

$$
\begin{aligned}
\left\{\mathfrak{F}\left(\mathcal{O}^{\prime}\right) \cap \mathfrak{F}\left(\tilde{\mathcal{O}}_{\mathfrak{M}_{k}}\right)\right]_{\pi_{0}}^{\prime \prime} \cap \mathscr{F}^{t} \cap \mathfrak{N}_{k} & =\mathscr{F}^{t}(\mathcal{O})_{\pi_{0}}^{\prime} \cap \mathscr{F}^{t} \cap \mathfrak{N}_{k} \\
& =\mathfrak{F}^{t}(\mathcal{O})^{c} \cap \mathfrak{N}_{k} .
\end{aligned}
$$

The second equation follows from the definition. To prove the first equation we note that

$$
\begin{aligned}
& \mathscr{F}^{t}(\mathcal{O})_{\pi_{0}}^{\prime} \cap \mathscr{F}^{t} \cap \mathfrak{N}_{k}=\mathscr{F}^{t}(\mathcal{O})^{c} \cap \mathscr{F}^{t} \cap \mathfrak{N}_{k} \\
& \subseteq \mathscr{F}^{t}(\mathcal{O})^{c} \cap \mathfrak{F}\left(\widetilde{\mathcal{O}}_{k+1}\right) \cap \mathscr{F}^{t} \subseteq\left[\mathscr{F}\left(\mathcal{O}^{\prime}\right) \cap \mathfrak{F}\left(\widetilde{\mathcal{O}}_{\mathfrak{M}_{k}}\right)\right]_{\pi_{0}}^{\prime \prime} \cap \mathscr{F}^{t},
\end{aligned}
$$

for some $\widetilde{\mathcal{O}}_{\mathfrak{R}_{k}}$, by condition (2) in the hypothesis. Thus, in the assertion, $\supseteq$ is proven, and because $\mathscr{F}\left(\mathcal{O}^{\prime}\right)_{\pi_{0}}^{\prime \prime}=\mathscr{F}^{t}(\mathcal{O})_{\pi_{0}}^{\prime}$ (twisted duality in $\pi_{0}$ ), the inclusion $\subseteq$ is trivial.

As we have already seen, every locally normal $*$-homorphism applied to $\mathfrak{N}_{k}$ is a *-isomorphism. Thus, $\pi \circ \pi_{0}^{-1}$ is a normal *-isomorphism for each $\mathfrak{N}_{k}$ and every ilngr $\pi$. We may therefore replace $\pi_{0}$ in (2.9) with an arbitrary ilngr $\pi$ of $\left(\mathscr{F}, \mathscr{G}, \alpha, \mathscr{F}^{t}\right)$. One has, then,

$$
m_{\pi}\left\{\left[\mathscr{F}\left(\mathcal{O}^{\prime}\right) \cap \tilde{\mathbb{F}}\left(\tilde{\mathcal{O}}_{\mathfrak{N}_{k}}\right)\right]_{\pi}^{\prime \prime} \cap \mathscr{F}^{t} \cap \mathfrak{N}_{k}\right\}=m_{\pi}\left\{\mathscr{F}^{t}(\mathcal{O})^{c} \cap \mathfrak{N}_{k}\right\} .
$$

Both sides are ascending sequences in $k$, and noting that $\mathfrak{F}\left(\tilde{\mathcal{O}}_{k+1}\right) \supset \mathfrak{N}_{k} \supset \mathfrak{F}\left(\tilde{\mathcal{O}}_{k}\right)$, Lemma 2.7 implies that the norm limit as $k \rightarrow \infty$ of the righthand side of $(2.10)$ is $m_{\pi}\left\{\widetilde{F}^{t}(\mathcal{O})^{c}\right\}$. As $\mathfrak{N}_{k} \subset \mathfrak{F}\left(\widetilde{\mathcal{O}}_{\mathfrak{N}_{k}}\right)$, it follows easily that the norm limit of the lefthand side of $(2.10)$ is

$$
\begin{aligned}
m_{\pi}\left\{\mathscr{F}\left(\mathcal{O}^{\prime}\right)_{\pi}^{\prime \prime} \cap \mathscr{F}^{t}\right\} & =m_{\pi}\left\{\mathscr{F}\left(\mathcal{O}^{\prime}\right)_{\pi}^{\prime \prime} \cap \mathscr{F}^{t} \cap \mathscr{A}\right\} \\
& =m_{\pi}\left\{\mathscr{F}\left(\mathcal{O}^{\prime}\right)_{\pi}^{\prime \prime} \cap \mathscr{A}\right\} .
\end{aligned}
$$

Thus, it follows that

$$
\left.\left.\overline{\left[m_{\pi}\left\{\mathscr{F}\left(\mathcal{O}^{\prime}\right)_{\pi}^{\prime \prime} \cap \mathscr{A}\right\}\right.}\right]_{\pi}^{w}=\overline{\left[m_{\pi}\left\{\tilde{F}^{t}(\mathcal{O})^{c}\right\}\right.}\right]_{\pi}^{w}
$$

The normality of $m_{\pi}$ permits us to take the weak limit inside of $m_{\pi}$, and Lemma 2.5 yields

$$
m_{\pi}\left\{\mathscr{F}\left(\mathcal{O}^{\prime}\right)_{\pi}^{\prime \prime}\right\}=m_{\pi}\left\{\mathscr{F}^{t}(\mathcal{O})_{\pi}^{\prime}\right\}
$$

so that

$$
\mathscr{A}\left(\mathcal{O}^{\prime}\right)_{\pi}^{\prime \prime}=m_{\pi}\left\{\mathfrak{F}(\mathcal{O})_{\pi}^{\prime}\right\}
$$

using (2.7) and the fact that $\mathfrak{F}^{t}(\mathcal{O})_{\pi}^{\prime}=\mathfrak{F}(\mathcal{O})_{\pi}^{\prime t}$. This completes the proof of Theorem 2.1.

Although it is duality for the observable algebras that is of most interest, we present a somewhat more restrictive set of conditions that yields twisted duality without the additional requirement that $\mathscr{G}$ be Abelian.

Theorem 2.8. Under the same assumptions as Theorem 2.1, but with condition (2) replaced by 
$\left(2^{\prime}\right)$ For any bdc's $\mathcal{O}, \mathcal{O}_{1}$ such that $\mathcal{O} \ll \mathcal{O}_{1}$ there exists a bdc $\mathcal{O}_{2}$ with $\mathcal{O}_{1} \ll \mathcal{O}_{2}$ such that

$$
\pi_{0}\left(\mathfrak{F}^{t}(\mathcal{O})^{e} \cap \mathfrak{F}\left(\mathcal{O}_{1}\right)\right) \subset\left[\mathscr{F}\left(\mathcal{O}^{\prime}\right) \cap \mathfrak{F}\left(\mathcal{O}_{2}\right)\right]_{\pi_{0}}^{\prime \prime},
$$

where $\mathscr{F}^{t}(\mathcal{O})^{e}=\mathscr{F}^{t}(\mathcal{O})^{\prime} \cap \mathscr{F}$, then $\pi\left(\mathscr{F}\left(\mathcal{O}^{\prime}\right)\right)^{\prime \prime}=\mathscr{F}^{t}(\mathcal{O})_{\pi}^{\prime}$ for every ilngr $\pi$ of $\left(\mathscr{F}, \mathscr{G}, \alpha, \mathscr{F}^{t}\right)$.

Remark. Although (2') is slightly stronger than (2), we show in Chap. IV that with the assumption (1), conditions (2) and (2') both hold for any net $\{\mathfrak{F}(\mathcal{O})\}$ such that additivity obtains (i.e. $\left.\left(\mathfrak{F}\left(\mathcal{O}_{1}\right) \bigvee \mathfrak{F}\left(\mathcal{O}_{2}\right)\right)^{\prime \prime}=\mathfrak{F}\left(\mathcal{O}_{1} \cup \mathcal{O}_{2}\right)\right)$ in the representation $\pi_{0}$.

Proof of Theorem 2.8. As this proof uses the same ideas as that of Theorem 2.1, we shall only indicate details concerning differences. First we note that, parallel to Lemma 2.4, we have

$$
\left(\mathfrak{M}_{n}^{e}\right)_{\pi}^{\prime \prime}=\left(\mathfrak{M}_{n}\right)_{\pi}^{\prime} \text { and }\left(\mathfrak{M}_{n}^{t e}\right)_{\pi}^{\prime \prime}=\left(\mathfrak{M}_{n}^{t}\right)_{\pi}^{\prime},
$$

for any $n$ and any ilngr $\pi\left(\mathfrak{M}_{n}^{e}=\mathfrak{M}_{n}^{\prime} \cap \mathscr{F}^{t}\right)$. The first equality in (2.11) follows because $\mathfrak{M}_{n}^{e} \supset \mathfrak{M}_{n}^{\prime} \cap \mathfrak{N}_{k}^{i}$ for all $k$. Thus, since the commutant theorem is valid within the type I factor $\mathfrak{N}_{k}^{t}$, a simple calculation shows that $\left(\left(\mathfrak{M}_{n} \cap \mathfrak{N}_{k}^{t}\right) \bigvee\left(\mathfrak{M}_{n}^{\prime} \cap \mathfrak{N}_{k}^{t}\right)\right)_{\mathfrak{M}_{k}}^{\prime \prime}=\mathfrak{N}_{k}^{t}$. Therefore, $\left(\mathfrak{M}_{n} \bigvee\left(\mathfrak{M}_{n}^{e}\right)_{\pi}^{\prime \prime}\right)_{\pi}^{\prime \prime}$ contains $\mathfrak{M}_{k}^{t}$ for all $k$. Thus, again, $\left(\mathfrak{M}_{n} \vee\left(\mathfrak{M}_{n}^{e}\right)_{\pi}^{\prime \prime}\right)_{\pi}^{\prime \prime}=\mathfrak{B}\left(\mathscr{H}_{\pi}\right)$ and $\mathfrak{M}_{n}$ type I implies $\left(\mathfrak{M}_{n}\right)^{\prime}=\left(\mathfrak{M}_{n}^{e}\right)^{\prime \prime}$. The proof of the second assertion in (2.11) is similar.

The parallel result to Lemma 2.5 is then, for any ilngr $\pi$ of $\left(\mathscr{F}, \mathscr{G}, \alpha, \mathscr{F}^{t}\right)$,

$$
\left(\mathfrak{F}(\mathcal{O})^{e}\right)_{\pi}^{\prime \prime}=\mathfrak{F}(\mathcal{O})_{\pi}^{\prime} \text { and }\left(\mathfrak{F}^{t}(\mathcal{O})^{e}\right)_{\pi}^{\prime \prime}=\mathfrak{F}^{t}(\mathcal{O})_{\pi}^{\prime} \text {, }
$$

proven as in Lemma 2 of [14], and the Lemma 2.6 becomes

$$
\mathfrak{F}(\mathcal{O})^{e}=\overline{\cup\left(\mathfrak{F}(\mathcal{O})^{e} \cap \mathfrak{N}_{k}^{t}\right)^{n}} \text { and } \mathfrak{F}^{t}(\mathcal{O})^{e}=\overline{\cup\left(\mathfrak{F}^{t}(\mathcal{O})^{e} \cap \mathfrak{N}_{k}\right)^{n}}
$$

(We briefly sketch the proof of the second equality in (2.12), assuming acquaintance with [14]. $A \in \mathbb{F}^{t}(\mathcal{O})^{e}$ is decomposed into odd and even parts $A_{ \pm}$(see (2.3)), as is the approximating $A_{\varepsilon} \in \mathfrak{F}\left(\tilde{\mathcal{O}}_{k}\right) \subset \mathfrak{N}_{k}: A_{\varepsilon}=A_{\varepsilon}^{+}+A_{\varepsilon}^{-}\left(\left\|A_{ \pm}-A_{\varepsilon}^{ \pm}\right\| \leqq \varepsilon\right)$, and the projection $P: \mathfrak{B}\left(\mathscr{H}_{\pi_{0}}\right) \rightarrow \mathfrak{N}_{k}: P(F)=P_{+}(F)+P_{-}(F), F \in \mathfrak{B}\left(\mathscr{H}_{\pi_{0}}\right)$. One shows $\| P_{ \pm}\left(A_{ \pm}\right)$ $-A_{\varepsilon}{ }^{ \pm} \| \leqq \varepsilon$ following [14]. Using assumption (1) in the hypothesis, the(anti) commutation relations in (2.3), and the faithfulness of $\pi_{0}$, one shows for any $A \in \mathfrak{F}^{t}(\mathcal{O})^{e}$, $B \in \mathfrak{F}(\mathcal{O})$, that $\left[A_{+}, B_{ \pm}\right]=0,\left[A_{-}, B_{+}\right]=0,\left[A_{-}, B_{-}\right]_{+}=0$. A straightforward calculation using these facts yields $P_{ \pm}\left(A_{ \pm}\right) \in \mathfrak{F}^{t}(\mathcal{O})^{e}$, so that $P_{ \pm}\left(A_{ \pm}\right) \in \mathfrak{F}^{t}(\mathcal{O})^{e} \cap \mathfrak{N}_{k}$ and $\left\|P_{+}\left(A_{+}\right)+P_{-}\left(A_{-}\right)-A\right\| \leqq 4 \varepsilon$. Now we shall not need to average over the gauge group. Indeed, we first prove the counterpart to (2.9): for every $k$, there exists a bdc $\widetilde{\mathcal{O}}_{\mathfrak{N}_{k}}$ so that

$$
\left[\mathscr{F}\left(\mathcal{O}^{\prime}\right) \cap \mathfrak{F}\left(\tilde{\mathcal{O}}_{\mathfrak{N}_{k}}\right)\right]_{\pi_{0}}^{\prime \prime} \cap \mathfrak{N}_{k}=\mathfrak{F}^{t}(\mathcal{O})_{\pi_{0}}^{\prime} \cap \mathfrak{N}_{k}=\mathfrak{F}^{t}(\mathcal{O})^{e} \cap \mathfrak{N}_{k},
$$

using condition $\left(2^{\prime}\right)$ and arguing as before. Again we may replace $\pi_{0}$ by any ilngr $\pi$ and use (2.12) to conclude directly that

$$
\mathscr{F}\left(\mathcal{O}^{\prime}\right)_{\pi}^{\prime \prime}=\mathscr{F}^{t}(\mathcal{O})_{\pi}^{\prime},
$$

completing the proof of Theorem 2.8 .

Remark. Because the $g_{0} \in \mathscr{G}$ in (2.3) is always in the stability group of the vacuum 
when one adds the usual relativistic assumptions [34], there is always a unitary $U\left(g_{0}\right)$ implementing the action of $\alpha_{g_{0}}(\cdot)$ in the representations occurring in relativistic quantum field theory. Thus, with the addition of these standard assumptions, one can omit the requirement in Theorem 2.8 that the representations be gauge representations.

\section{Normal Product States for Free Fermion Field Algebras}

In this chapter we shall establish properties for the free Fermi field algebra that shall

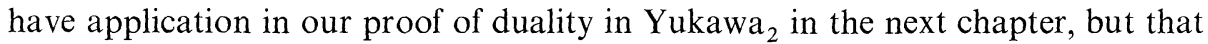
have some intrinsic interest as well. As previously mentioned, the primary result will be that any pair of normal states $\phi_{1}$ of $\mathfrak{F}\left(\mathcal{O}_{1}\right)$ and $\phi_{2}$ of $\mathfrak{F}^{t}\left(\mathcal{O}_{2}\right), \mathcal{O}_{1}$ and $\mathcal{O}_{2}$ spacelike separated regions in two-dimensional (or three- or four-dimensional) space-time and $\mathfrak{F}\left(\mathcal{O}_{i}\right)$ the free fermion algebra (on Fock space $\mathscr{F}_{f}$ ) associated with the region $\mathcal{O}_{i}$, will have an extension to a normal state $\phi^{\prime}$ of the algebra generated by $\mathfrak{F}\left(\mathcal{O}_{1}\right)$ and $\mathfrak{F}^{t}\left(\mathcal{O}_{2}\right)$ which is a product state for $\mathfrak{F}\left(\mathcal{O}_{1}\right)$ and $\mathfrak{F}^{t}\left(\mathcal{O}_{2}\right)$, i.e. for all $A_{n} \in \mathfrak{F}\left(\mathcal{O}_{1}\right), B_{n} \in \mathfrak{F}^{t}\left(\mathcal{O}_{2}\right)$, $\phi^{\prime}\left(\sum A_{n} B_{n}\right)=\sum \phi_{1}\left(A_{n}\right) \phi_{2}\left(B_{n}\right)$. We remark that if the restriction that $\phi^{\prime}$ be a normal state is removed above, the result holds in any standard Wightman-type field theory [37], and is an expression of the statistical independence of algebras associated with spacelike separated regions $[24,37]$. From the existence of normal product states one can conclude a number of interesting results (see [8]), among them being the funnel property defined in Chap. II. As our proof of the existence of normal product states for the free Fermi field shall parallel, to a large extent, that of Buchholz [8] for the free Bose field, we shall, for the benefit of the reader, keep our notation as close as possible to his.

We must begin to recall a few concepts and introduce notation. If $K$ is a separable (complex) Hilbert space, $\mathscr{A}(K)$ - the CAR algebra over $K$ - is the $C^{*}$ algebra generated by elements $a(f)$, where $f \rightarrow a(f)$ is a linear map of $K$ into $\mathscr{A}(K)$ satisfying the canonical anticommutation relations; specifically, for $f, g \in K$,

$$
\begin{aligned}
a(f)^{*} a(g)+a(g) a(f)^{*} & =\langle f, g\rangle I, \\
a(f) a(g)+a(g) a(f) & =0,
\end{aligned}
$$

where $\langle\cdot, \cdot\rangle$ is the inner product on $K$ and $I$ is the unit operator in $\mathscr{A}(K)$.

The gauge-invariant generalized (or quasi-) free states of $\mathscr{A}(K)$ are states $\phi_{A}$ whose $n$-point functions have the structure

$$
\phi_{A}\left(a\left(f_{n}\right)^{*} \ldots a\left(f_{1}\right)^{*} a\left(g_{1}\right) \ldots a\left(g_{m}\right)=\delta_{n m} \operatorname{det}\left(\left\langle f_{i}, A g_{j}\right\rangle\right),\right.
$$

for all $f, g \in K$, where $A$ is a linear operator on $K$ such that $0 \leqq A \leqq I$. These states have been studied in some depth (see $[44,32]$ and references there) and shall be used as tools here. Because the GNS representation $\left(\mathscr{H}_{A}, \pi_{A}, U_{A}\right)$ associated with such a state is covariant (with respect to the group $\mathscr{G}_{0}=\left\{e, g_{0}\right\}$ (the multiplicative group on two elements $\{1,-1\}$ ), where $\alpha_{g_{0}}(a(f))=a(-f)$ ), there exists a representation $\rho_{A}$ of the covariance algebra $\mathscr{A}(K) \otimes_{\alpha} \mathscr{G}_{0}$ on $\mathscr{H}_{A}$. Since $\mathscr{A}(K) \otimes_{\alpha} \mathscr{G}_{0}$ is simple (follows from Theorems 8.11.12,8.10.12 and 8.1.9 in [50], since $\mathscr{A}(K)$ is simple, $\mathscr{G}_{0}$ is Abelian and discrete, and the fixed point subalgebra of $\mathscr{A}(K)$ under $\left\{\mathscr{G}_{0}, \alpha\right\}$ is simple- see also [47] for relevant remarks), this representation is in fact a $*$-isomorphism. The state 
$\phi_{A}$ extends canonically to a state $\psi_{A}$ on $\mathscr{A}(K) \otimes_{\alpha} \mathscr{G}_{0}$. If $K=K_{1} \oplus K_{2}$ and $\mathscr{E}(K)$ is the $C^{*}$-algebra generated by $\mathscr{A}\left(K_{1}\right)$ and $\mathscr{A}^{t}\left(K_{2}\right)$ in the covariance algebra, where the superscript $t$ represents a twist (2.5) determined by the unitary operator $U_{A}\left(g_{0}\right)$ implementing $\alpha_{g_{0}}$, it is easy to see that the restriction of $\psi_{A}$ to $\mathscr{E}(K)$ defines a state $\phi_{A}^{\prime}$ whose $n$-point functions are of the form

$$
\begin{aligned}
\phi_{A}^{\prime}\left(\left(a\left(f_{n}^{(1)}\right)^{*}\right.\right. & \left.\left.+a^{t}\left(f_{n}^{(2)}\right)^{*}\right) \ldots\left(a\left(f_{1}^{(1)}\right)^{*}+a^{t}\left(f_{1}^{(2)}\right)^{*}\right)\left(a\left(g_{1}^{(1)}\right)+a^{t}\left(g_{1}^{(2)}\right)\right) \ldots\left(a\left(g_{m}^{(1)}\right)+a^{t}\left(g_{m}^{(2)}\right)\right)\right) \\
& =\delta_{n m} \operatorname{det}\left(\left\langle f_{i}^{\prime}, A g_{j}^{\prime}\right\rangle\right)\left(=\phi_{A}\left(a\left(f_{n}^{\prime}\right)^{*} \ldots a\left(f_{1}^{\prime}\right)^{*} a\left(g_{1}^{\prime}\right) \ldots a\left(g_{m}^{\prime}\right)\right)\right),
\end{aligned}
$$

where $f_{j}^{\prime}=f_{j}^{(1)}+(-1)^{n-j+1}$ if $f_{j}^{(2)}$ (similarly for $g_{j}^{\prime}$ ) and $f_{j}=f_{j}^{(1)}+f_{j}^{(2)}, f_{j}^{(i)} \in K_{i}$.

In two space-time dimensions the free Fermi field is given by [17]

$$
\begin{aligned}
\Psi(x, t) & =\left\{\begin{array}{l}
\Psi^{(1)}(x, t) \\
\Psi^{(2)}(x, t)
\end{array}\right\} \\
& =(4 \pi)^{-1 / 2} \int e^{-i p x}\left(e^{i \omega t} b^{\prime}(p)^{*}\left\{\begin{array}{c}
v(-p) \\
v(p)
\end{array}\right\}+e^{-i \omega t} b(-p)\left\{\begin{array}{c}
v(p) \\
-v(-p)
\end{array}\right\}\right) \omega(p)^{-1 / 2} d p,
\end{aligned}
$$

where $\omega(p)=\left(p^{2}+m_{f}^{2}\right)^{1 / 2}, v(p)=(\omega(p)+p)^{1 / 2}, p$ denotes the spatial component of the energy-momentum spectrum vector, $m_{f}>0$ is the fermion mass. $\Psi(x, t)$ is a distribution taking values in $\mathfrak{B}\left(\mathscr{F}_{f}\right)$, where $\mathscr{F}_{f}$ is the standard relativistic Fermi Fock space. The annihilation and creation operators $b^{\prime}(p)^{(*)}, b(p)^{(*)}$, satisfy the usual anticommutation relations, so that, as distributions,

$$
\begin{aligned}
& {\left[\Psi^{(i)}(x, t), \Psi^{(j)}(y, t)^{*}\right]_{+}} \\
& \quad \equiv \Psi^{(i)}(x, t) \Psi^{(j)}(y, t)^{*}+\Psi^{(j)}(y, t)^{*} \Psi^{(i)}(x, t)=\delta(x-y) \delta_{i j}
\end{aligned}
$$

(all other anticommutators vanish). Further, one requires (Fock representation)

$$
b(p) \Omega_{0}=0=b^{\prime}(p) \Omega_{0},
$$

where $\Omega_{0}$ is the (unique) vacuum (translation-invariant) vector. Defining $B(F)=$ $\Psi_{0}^{(1)}\left(f^{(1)}\right)+\Psi_{0}^{(2)}\left(f^{(2)}\right), \quad F=\left(f^{(1)}, f^{(2)}\right) \in L_{2}(\mathbb{R}) \oplus L_{2}(\mathbb{R}), \quad \Psi_{0}^{(i)}\left(f^{(i)}\right)=\int \Psi^{(i)}(x, t=0) \times$ $f^{(i)}(x) d x$, one notes

$$
\begin{aligned}
{\left[B(F)^{*}, B(G)\right]_{+} } & =\left\{\left\langle f^{(1)}, g^{(1)}\right\rangle_{L_{2}(\mathbb{P})}+\left\langle f^{(2)}, g^{(2)}\right\rangle_{L_{2}(\mathbb{R})}\right\} I \\
& =\langle F, G\rangle_{L_{2}(\mathbb{R})^{\oplus 2} . I .}
\end{aligned}
$$

Denoting by $\phi_{0}(\cdot)$ the expectation given by $\left\langle\Omega_{0}, \Omega_{0}\right\rangle$, one easily sees that

$$
\phi_{0}\left(B(F)^{*} B(G)\right)=\frac{1}{2}\left\langle F,\left(\begin{array}{cc}
C_{-} & 0 \\
0 & C_{+}
\end{array}\right)\left(\begin{array}{ll}
1 & 1 \\
1 & 1
\end{array}\right)\left(\begin{array}{cc}
C_{-} & 0 \\
0 & C_{+}
\end{array}\right) G\right\rangle_{L_{2}(\mathbb{R})^{\oplus^{2}}},
$$

where $C_{ \pm}$is the operator whose Fourier transform is given by $[1 \pm(p / \omega(p))]^{1 / 2}$. As a quasi-free state, $\phi_{0}$ is completely determined by this two-point function. The local CAR algebra $\mathscr{A}(K(\underline{\mathcal{O}})$ ) (whose weak closure defines the time-zero free Fermi field algebra $\mathfrak{F}(\underline{\mathcal{O}}))$ associated with region $\mathcal{Q} \subset \mathbb{R}(t=0)$ is defined as the $C^{*}$-algebra generated by the field operators $B(F)$ with test functions $F \in K(\underline{\mathcal{O}})$, where $K(\underline{\mathcal{O}})=$ $\mathscr{S}(\underline{\mathcal{O}}) \oplus \mathscr{S}(\underline{\mathcal{O}}) ; \mathscr{S}(\underline{\mathcal{O}})$ denotes the subset of $\mathscr{S}(\mathbb{R})$ (the set of all rapidly decreasing $C^{\infty}$ functions) with support in $\underline{\mathcal{O}}$. One knows [17] that the local ring $\mathfrak{F}(\mathcal{O})$, generated by the field $\Psi(x, t)$ smeared with test functions with support in $\mathcal{O} \subset \mathbb{R}^{2}$, is the weak 
closure of $\mathscr{A}(K(\underline{O}))$ if $\mathcal{O}$ is the double cone with base $\underline{Q}$ at time $t=0$. Denoting with $N$ the number operator on $\mathscr{F}_{f}$, we define the twist in $(2.5)$ by choosing $Z=(1-i) / 2$ $+(1+i) e^{i \pi N} / 2$. One verifies easily that $(2.6)$ obtains with this definition.

Supplying $\widetilde{F}_{f}$ with the standard unitary representation of the Lorentz group and verifying the Wightman axioms [46], one knows that the vacuum $\Omega_{0}$ is cyclic and separating for each $\mathscr{F}(\mathcal{O})$ (or $\mathscr{A}\left(K(\underline{\mathcal{O}})\right.$ )) such that the spacelike complement $\mathcal{O}^{\prime}$ is nonempty [46]. And $\mathfrak{F}(\mathcal{O})$ satisfies, for every $\mathcal{O}_{1}$ such that $\mathcal{O} \ll \mathcal{O}_{1}, A B=0$ for $A \in \mathfrak{F}(\mathcal{O})$ and $B \in \mathfrak{F}\left(\mathcal{O}_{1}\right)^{\prime}$ implies $A=0$ or $B=0$ (proven easily using [45]). Thus, from [8], one has:

Theorem 3.1. [8]. Let $\hat{\mathcal{O}}_{1}$ and $\hat{\mathcal{O}}_{2}$ be two spacelike separated regions such that $\mathcal{O}_{1}+\mathscr{N} \subset \hat{\mathcal{O}}_{1}$ and $\mathcal{O}_{2}+\mathscr{N} \subset \hat{\mathcal{O}}_{2}$, for some neighbourhood $\mathscr{N}$ of the origin in $\mathbb{R}^{d}$ and $\mathscr{O}_{1}, \mathcal{O}_{2}$, spacelike separated subsets of $\mathbb{R}^{d}$.

(1) If there exists a normal product state for $\mathfrak{F}\left(\hat{\mathcal{O}}_{1}\right)$ and $\mathfrak{F}^{t}\left(\hat{\mathcal{O}}_{2}\right)$, then every pair of normal states $\phi_{1}$ of $\mathfrak{F}\left(\mathcal{O}_{1}\right)$ and $\phi_{2}$ of $\mathfrak{F}^{t}\left(\mathcal{O}_{2}\right)$ can be extended to a normal product state $\phi^{\prime}$ of $\mathfrak{F}\left(\mathcal{O}_{1}\right)$ and $\mathfrak{F}^{t}\left(\mathcal{O}_{2}\right)$.

(2) There exist factors of type $1, \mathfrak{M}_{1}$ and $\mathfrak{M}_{2}$, such that $\mathfrak{F}\left(\mathbb{O}_{1}\right) \subset \mathfrak{M}_{1} \subset \mathfrak{M}_{2}^{\prime} \subset$ $\mathfrak{F}^{t}\left(\mathcal{O}_{2}\right)^{\prime}$, if (and only if) there exist normal product states for $\mathfrak{F}\left(\mathcal{O}_{1}\right)$ and $\mathfrak{F}^{t}\left(\mathcal{O}_{2}\right)$.

By (2) and twisted duality for the free Fermi field, given any bounded double cones $\mathcal{O}_{1} \ll \mathcal{O}_{3}$ (in two or four space-time dimensions) such that there exists a normal product state for $\mathfrak{F}\left(\mathcal{O}_{1}\right)$ and $\mathscr{F}^{t}\left(\mathcal{O}_{3}^{\prime}\right)$, there exists a type I factor $\mathfrak{M}$ such that

$$
\mathfrak{F}\left(\mathcal{O}_{1}\right) \subset \mathfrak{M} \subset \mathscr{F}^{t}\left(\mathcal{O}_{3}^{\prime}\right)^{\prime}=\mathfrak{F}\left(\mathcal{O}_{3}\right),
$$

which gives the funnel property for the free Fermi field algebra.

In order to show that there exist normal product states for $\mathfrak{F}\left(\mathcal{O}_{1}\right)$ and $\mathscr{F}^{t}\left(\mathcal{O}_{3}^{\prime}\right)$, $\mathcal{O}_{1} \ll \mathcal{O}_{3}$, we proceed as in [8]. From Theorem 3.1, (1), it will suffice to verify that there exists a normal product state for $\mathfrak{F}\left(\hat{\mathcal{O}}_{1}\right)$ and $\mathscr{F}^{t}\left(\hat{\mathcal{O}}_{3}^{\prime}\right)$ which coincides with the Fock vacuum state $\phi_{0}$ on each algebra separately. To fix notation, $\underline{Q}_{1} \subset \mathbb{R}$ will be presumed to be bounded ( $\hat{\mathcal{O}}_{1}$ will be contained in its causal shadow) and $\underline{\mathcal{O}}_{2} \subset$ $\mathbb{R}\left(\hat{\mathcal{O}}_{3}^{\prime}\right.$ will be contained in its causal shadow) will have compact complement. The distance between $\underline{O}_{1}$ and $\underline{O}_{2}$ is assumed strictly greater than zero. All interesting choices of $\mathcal{O}_{1}$ and $\mathcal{O}_{2}$ can be reduced to this case by making a Lorentz transformation.

We define a positive functional $\phi_{p}^{\prime}$ on $\mathfrak{F}\left(\underline{\mathcal{O}}_{1}\right) \vee \mathfrak{F}^{t}\left(\underline{\Theta}_{2}\right)$ (the algebra of all finite sums $\sum A_{n} B_{n}, A_{n} \in \mathfrak{F}\left(\underline{O}_{1}\right)$ and $\left.B_{n} \in \mathfrak{F}^{t}\left(\underline{\mathcal{O}}_{2}\right)\right)$ by

$$
\phi_{p}^{\prime}\left(\sum A_{n} B_{n}\right)=\sum \phi_{0}\left(A_{n}\right) \phi_{0}\left(B_{n}\right)
$$

It is clear that $\phi_{p}^{\prime}$ induces a representation of the algebra $\mathscr{E}(K(\underline{\mathcal{O}})) \equiv \overline{\mathscr{A}\left(K\left(\underline{Q}_{1}\right)\right) \mathrm{V}}$ $\overline{\mathscr{A}^{t}\left(K\left(\underline{O}_{2}\right)\right)^{n}}\left(\underline{O} \equiv \underline{\mathcal{O}}_{1} \cup \underline{\mathcal{O}}_{2}\right)$, and we denote the corresponding state again $\phi_{p}^{\prime}$. A straightforward calculation shows that the states $\phi_{p}^{\prime}$ and $\phi_{0}^{\prime}$ on $\mathscr{E}(K(\underline{\mathcal{O}}))$ (the latter given by the expectation $\left.\left\langle\Omega_{0}, \Omega_{0}\right\rangle\right)$ are of the form (3.1). Extending them to states

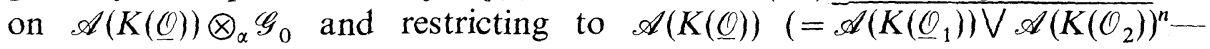
see proof of Prop. 4.1) defines gauge-invariant quasifree states $\phi_{p}$ and $\phi_{0}$ on $\mathscr{A}(K(\underline{\mathcal{O}}))$. In fact, as every $B_{n} \in \mathfrak{F}^{t}\left(\underline{\mathcal{O}}_{2}\right)$ is of the form $Z C_{n} Z^{*}, C_{n} \in \mathfrak{F}\left(\underline{\mathcal{O}}_{2}\right)$,

$$
\sum \phi_{0}\left(A_{n}\right) \phi_{0}\left(B_{n}\right)=\sum \phi_{0}\left(A_{n}\right) \phi_{0}\left(C_{n}\right)=\phi_{p}\left(\sum A_{n} C_{n}\right)
$$


Since, by definition, $\phi_{0}^{\prime}$ is a normal (density matrix) state on $\mathscr{E}(K(\underline{O})$ ) (it is a vector state in Fock space), if we show that the representations of $\mathscr{E}(K(\underline{O}))$ induced by $\phi_{p}^{\prime}$ and $\phi_{0}^{\prime}$ are unitarily equivalent, the normality of $\phi_{p}^{\prime}$ follows at once. (The same remark is valid for $\phi_{0}$ and $\phi_{p}$ on $\mathscr{A}(K(\underline{\mathcal{O}}))$.) In an earlier version of this paper we asserted that the normality of $\phi_{p}$ on $\mathscr{A}(K(\underline{\mathcal{O}}))$ implies the normality of $\phi_{p}^{\prime}$ on $\mathscr{E}(K(\underline{\mathcal{O}}))$, and although this is true, the proof is more lengthy than we at first thought. Thus, here, we prove the normality of $\phi_{p}$ and $\phi_{p}^{\prime}$ on their respective algebras independently. Before we do that, we must introduce some further notation.

Let $K_{0}(\underline{\mathcal{O}})$ be the completion of $K(\underline{\mathcal{O}})$ in the $L_{2}(\mathbb{R})^{\oplus 2}$ norm (call it $\left.\|\cdot\|_{0}\right)$. If $E$ is the orthogonal projection in $L_{2}(\mathbb{R}) \oplus L_{2}(\mathbb{R})$ onto $K_{0}(\underline{\mathcal{O}})$, the two-point function of $\phi_{0} \uparrow \mathscr{A}\left(K_{0}(\mathcal{O})\right)$ is

$$
\phi_{0}\left(B(F)^{*} B(G)\right)=\left\langle F, S_{0} G\right\rangle_{L_{2}(\mathbb{P})^{\oplus^{2}}}
$$

with $S_{0}=\frac{1}{2} E \mathscr{C}\left(\begin{array}{ll}1 & 1 \\ 1 & 1\end{array}\right) \mathscr{C} E$ a positive operator bounded by 1 and $\mathscr{C}$ is the bounded operator $\left(\begin{array}{cc}C_{-} & 0 \\ 0 & C_{+}\end{array}\right)$. Every $F \in K(\underline{\Theta})$ can be decomposed uniquely into a sum $F=F_{1}+F_{2}, F_{i} \in K\left(\underline{\mathcal{O}}_{2}\right) i=1,2$. Thus, from (3.2),

$$
\phi_{p}\left(B(F)^{*} B(G)\right)=\phi_{0}\left(B\left(F_{1}\right)^{*} B\left(G_{1}\right)\right)+\phi_{0}\left(B\left(F_{2}\right)^{*} B\left(G_{2}\right)\right)
$$

is the two-point function ( $\phi_{p}$ is clearly a quasifree state). Defining $\langle F, G\rangle_{p}=$ $\left\langle F_{1}, G_{1}\right\rangle_{L_{2}(\mathbb{R})^{\oplus 2}}+\left\langle F_{2}, G_{2}\right\rangle_{L_{2}(\mathbb{R})^{\oplus 2}}$, we denote by $K_{p}(\underline{\mathcal{O}})$ the completion of $K(\underline{\mathcal{O}})$ in $\|\cdot\|_{p}$. Due to the decomposition $F=F_{1}+F_{2}$ already mentioned there exist two mutually orthogonal projections $\Theta_{1}, \Theta_{2}$, on $K_{p}(\underline{\mathcal{O}})$ such that $\Theta_{i} F=F_{i}, i=1,2$, for every $F \in K(\underline{\mathcal{O}})$. Thus, $K_{p}(\underline{\mathcal{O}})=K_{0}\left(\underline{\mathcal{O}}_{1}\right) \oplus K_{0}\left(\underline{\mathcal{O}}_{2}\right)$ and $\phi_{p}\left\lceil\mathscr{A}\left(K_{p}(\underline{\mathcal{O}})\right)\right.$ is determined by

where

$$
\phi_{p}\left(B(F)^{*} B(G)\right)=\left\langle F, S_{p} G\right\rangle_{p},
$$

$$
S_{p}=\frac{1}{2} E_{1} \mathscr{C}\left(\begin{array}{ll}
1 & 1 \\
1 & 1
\end{array}\right) \mathscr{C} E_{1} \Theta_{1}+\frac{1}{2} E_{2} \mathscr{C}\left(\begin{array}{ll}
1 & 1 \\
1 & 1
\end{array}\right) \mathscr{C} E_{2} \Theta_{2}
$$

is a positive operator with bound 1 and $E_{i}$ is the orthogonal projection in $L_{2}(\mathbb{R}) \oplus L_{2}(\mathbb{R})$ onto $K_{0}\left(\underline{O}_{i}\right), i=1,2$.

Noting that $K_{0}(\underline{\mathcal{O}})=K_{p}(\underline{\mathcal{O}})=L_{2}(\underline{\mathcal{O}}) \oplus L_{2}(\underline{\mathcal{O}})$, we may drop the operators $\Theta_{i}$. Therefore, we must compare two gauge-invariant representations of the CAR over the same Hilbert space. Through (3.1) the operators above determine $\phi_{0}^{\prime} \uparrow \mathscr{E}\left(K_{0}(\underline{\mathcal{O}})\right)$ and $\phi_{p}^{\prime} \uparrow \mathscr{E}\left(K_{p}(\underline{O})\right)$. (Note that due to the norm continuity of the generators of $\mathscr{E}$, $\mathscr{E}(K(\underline{\mathcal{O}}))=\mathscr{E}\left(K_{0}(\underline{\mathcal{O}})\right)=\mathscr{E}\left(K_{p}(\underline{\mathcal{O}})\right)$, just as $\mathscr{A}(K(\underline{\mathcal{O}}))=\mathscr{A}\left(K_{0}(\underline{\mathcal{O}})\right)=\mathscr{A}\left(K_{p}(\underline{\mathcal{O}})\right)$.)

Theorem 3.2. If the following conditions hold:

1) $S_{0}$ and $S_{p}$ do not have eigenvalues 0 and 1 on $L_{2}(\underline{O})^{\oplus 2}$,

2) $S_{0}-S_{p}$ is a trace class operator on $L_{2}(\underline{\mathcal{O}})^{\oplus 2}$, then we have

a) the representations $\pi_{0}^{\prime}$ and $\pi_{p}^{\prime}$ of $\mathscr{E}(K(\underline{\mathcal{O}}))$ induced by $\phi_{0}^{\prime}$ and $\phi_{p}^{\prime}$, respectively, are unitarily equivalent. 
b) the representations $\pi_{0}$ and $\pi_{p}$ of $\mathscr{A}(K(\underline{\mathcal{O}}))$ induced by $\phi_{0}$ and $\phi_{p}$, respectively, are unitarily equivalent.

Remark. With $S_{0}$ and $S_{p}$ defined as the unique positive operators that determine the vacuum state $\phi_{0}\left\lceil\mathscr{A}(K(\underline{\mathcal{O}}))\right.$ and the product state $\phi_{p}=\phi_{0} \uparrow \mathscr{A}\left(K\left(\underline{\mathcal{O}}_{1}\right)\right) \times$ $\phi_{0}\left\lceil\mathscr{A}\left(K\left(\underline{O}_{2}\right)\right)\right.$ in the manner indicated in (3.1), this theorem is valid if one chooses $\underline{\mathcal{O}} \equiv \underline{\mathcal{O}}_{1} \cup \underline{\mathcal{O}}_{2} \subset \mathbb{R}^{d-1}, d=2,3,4$, i.e. the proof is independent of the number of spacetime dimensions.

Proof. That (1) and (2) imply (b) follows from [51,32]. We shall use arguments of [51] to prove (a) and (b) essentially simultaneously. Let $K=L_{2}(\underline{\mathcal{O}})^{\oplus 2}$ and $K_{i}=$ $L_{2}\left(\underline{Q}_{i}\right)^{\oplus 2}, i=1,2$. Let $a(f)$ and $b^{*}(f)$ (both linear in $f$ ) be two independent Fock representations of the CAR algebra over $K$ with a common Fock vector $\Omega_{0}$. That is, we require

$$
\begin{gathered}
{[a(f), a(g)]_{+}=\left[b^{*}(f), b^{*}(g)\right]_{+}=\left[a(f), b^{*}(g)\right]_{+}=[a(f), b(g)]_{+}=0,} \\
{\left[a(f), a^{*}(g)\right]_{+}=\left[b(g), b^{*}(f)\right]_{+}=\langle g, f\rangle,} \\
a(f) \Omega_{0}=b(g) \Omega_{0}=0,
\end{gathered}
$$

for every $f, g \in K$. $\mathscr{H}$ will denote the associated Fock space. If $H$ and $L$ are operators in $\mathfrak{B}(K)$ satisfying $H^{*} H+L^{*} L=I$, then setting $A(f)=a(H f)+b^{*}(L f), f \in K$, defines again a representation of the algebra $\mathscr{A}(K)$. Further, if 0 is not an eigen value for $L^{*}$ or $H^{*} \Omega_{0}$ is cyclic for $\left\{A(f), A^{*}(f) \mid f \in K\right\}$ (Theorem 1 [51]). Moreover,

$$
\left\langle\Omega_{0}, A^{*}\left(f_{n}\right) \ldots A^{*}\left(f_{1}\right) A\left(g_{1}\right) \ldots A\left(g_{m}\right) \Omega_{0}\right\rangle=\delta_{n m} \operatorname{det}\left(\left\langle f_{i}, L^{*} L g_{j}\right\rangle\right) .
$$

Thus, choosing $L=S_{0}^{1 / 2}$ and $H=\left(I-S_{0}\right)^{1 / 2}$ (or with $S_{0}$ replaced by $S_{p}$ in both operators), the corresponding representation $\left\{A(f), \mathscr{H}, \Omega_{0}\right\}$ is unitarily equivalent to the GNS representation of $\mathscr{A}(K)$ induced by the state $\phi_{0}\left(\phi_{p}\right)$.

Let $N_{K}$ denote the number operator on $\mathscr{H}$ and define $U\left(g_{0}\right)=e^{i \pi N_{K}}$, $Z_{K}=(1-i) / 2+(1+i) e^{\imath \pi N_{K}} / 2 . \quad$ Set $\quad \mathscr{A}(f)=A\left(f^{(1)}\right)+A^{t}\left(f^{(2)}\right), \quad f \in K, \quad f=$ $f^{(1)}+f^{(2)}, f^{(i)} \in K_{i}$, where the twist $t$ is defined using this $Z$. It is easy to see that the $C^{*}$-algebra generated by $\{\mathscr{A}(f) \mid f \in K\}$ is isomorphic to $\mathscr{E}(K)$, and because $U\left(g_{0}\right) \Omega_{0}=\Omega_{0}\left(N_{K} \Omega_{0}=0\right)$, a straightforward calculation shows that

$$
\left\langle\Omega_{0}, \mathscr{A}^{*}\left(f_{n}\right) \ldots \mathscr{A}^{*}\left(f_{1}\right) \mathscr{A}\left(g_{1}\right) \ldots \mathscr{A}\left(g_{m}\right) \Omega_{0}\right\rangle=\delta_{n m} \operatorname{det}\left(\left\langle f_{i}^{\prime}, L^{*} L g_{j}^{\prime}\right\rangle\right),
$$

where $f_{j}^{\prime}$ and $g_{j}^{\prime}$ are given as in (3.1). A simple argument shows that any even vector (i.e. invariant under $\left.U\left(g_{0}\right)\right)$ that is cyclic for $\left\{A(f), A^{*}(f) \mid f \in K\right\}$ is cyclic for $\{\mathscr{A}(f)$, $\left.\mathscr{A}^{*}(f) \mid f \in K\right\}$ (use $A^{t}\left(f^{(2)}\right)=A\left(-i f^{(2)}\right) U\left(g_{0}\right)=U\left(g_{0}\right) A\left(i f^{(2)}\right)$ ). Therefore, choosing $L$ and $H$ as above, the corresponding representation $\left\{\mathscr{A}_{0}(f), \mathscr{H}, \Omega_{0}\right\}\left(\left\{\mathscr{A}_{p}(f)\right.\right.$, $\left.\mathscr{H}, \Omega_{0}\right\}$ ) is unitarily equivalent to the GNS representation of $\mathscr{E}(K)$ induced by $\phi_{0}^{\prime}\left(\phi_{p}^{\prime}\right)$.

Now consider $\bar{H}$ and $\bar{L}$ in $\mathfrak{B}(K)$ such that

$$
\begin{gathered}
\bar{H}^{*} \bar{H}+\bar{L}^{*} \bar{L}=I=\bar{H} \bar{H}^{*}+\bar{L} \bar{L}^{*}, \\
\bar{H}^{*} \bar{L}=\bar{L}^{*} \bar{H}, \bar{H} \bar{L}^{*}=\bar{L} \bar{H}^{*} .
\end{gathered}
$$


Then one knows (Lemma 1 [51]) that if $\bar{L}$ is Hilbert-Schmidt, the vector

$$
\Omega \equiv \prod_{i}\left(\sqrt{1-\lambda_{i}^{2}}-\varepsilon_{i} \lambda_{i} a^{*}\left(g_{i}\right) b^{*}\left(g_{i}\right)\right) \Omega_{0}=\prod_{i}\left(\sqrt{1-\lambda_{i}^{2}}-\varepsilon_{i} \lambda_{i} a^{t *}\left(g_{i}\right) b^{t *}\left(g_{i}\right)\right) \Omega_{0}
$$

is in $\mathscr{H}$, where $\left\{g_{i}\right\}$ is an orthonormal basis in $K, \varepsilon_{i}= \pm 1$, and the $\lambda_{i}$ 's are eigenvalues of $|\bar{L}|$. The equality above follows from the fact that $Z_{K} a^{*}\left(g_{i}\right) b^{*}\left(g_{i}\right) Z_{K}^{*}=a^{*}\left(g_{i}\right) b^{*}\left(g_{i}\right)$. This is a very special vector, because defining

$$
A(f)=a(\bar{H} f)+b^{*}(\bar{L} f) \text { and } B^{*}(f)=-a(\bar{L} f)+\mathrm{b}^{*}(\bar{H} f),
$$

$A(f)$ and $B^{*}(f)$ are two anticommuting fields that are irreducible in $\mathscr{H}$ (see Lemma 1 [51]), and $A(f) \Omega=B(g) \Omega=0$, for all $f, g \in K$. Because $\Omega$ is even, one has $\mathscr{A}(f) \Omega=\mathscr{B}(g) \Omega=0$, for all $f, g \in K$, where

$$
\mathscr{A}(f)=A\left(f^{(1)}\right)+A^{t}\left(f^{(2)}\right), \mathscr{B}^{*}(g)=B^{*}\left(g^{(1)}\right)+B^{* t}\left(g^{(2)}\right) .
$$

Since $\{A(f), B(f) \mid f \in K\}$ generates an irreducible *algebra, $\Omega$ is cyclic for this algebra and, therefore, for the *-algebra generated by $\{\mathscr{A}(f) \mathscr{B}(f) \mid f \in K\}$. Therefore, setting

$$
\begin{gathered}
W \Omega_{0}=\Omega, \\
W \prod_{i=1}^{n}\left(a^{*}\left(f_{i}^{(1)}\right)+a^{* t}\left(f_{i}^{(2)}\right)\right) \prod_{j=1}^{m}\left(b^{*}\left(g_{j}^{(1)}\right)+b^{* t}\left(g_{j}^{(2)}\right)\right) \Omega_{0} \\
=\prod_{i=1}^{n} \mathscr{A}^{*}\left(f_{i}\right) \prod_{j=1}^{m} \mathscr{B}^{*}\left(g_{j}\right) \Omega,
\end{gathered}
$$

$W$ defines a linear isometric ( $\|\Omega\|=1$ ) operator with dense domain and range; thus, it defines a unitary operator (call it $W$ again) such that

$$
\begin{array}{ll}
W\left(a\left(f^{(1)}\right)+a^{t}\left(f^{(2)}\right)\right) W^{*}=\mathscr{A}(f), & \forall f \in K, \\
\left.W(b)\left(f^{(1)}\right)+b^{t}\left(f^{(2)}\right)\right) W^{*}=\mathscr{B}(f), & \forall f \in K .
\end{array}
$$

We return to our representations $\left\{\mathscr{A}_{0(p)}(f), \mathscr{H}, \Omega_{0}\right\}$ and recall

$$
\begin{aligned}
& \mathscr{A}_{0}(f)=a\left(\left(I-S_{0}\right)^{1 / 2} f^{(1)}\right)+a^{t}\left(\left(I-S_{0}\right)^{1 / 2} f^{(2)}\right)+b^{*}\left(S_{0}^{1 / 2} f^{(1)}\right)+b^{* t}\left(S_{0}^{1 / 2} f^{(2)}\right), \\
& \mathscr{A}_{p}(f)=a\left(\left(I-S_{p}\right)^{1 / 2} f^{(1)}\right)+a^{t}\left(\left(I-S_{p}\right)^{1 / 2} f^{(2)}\right)+b^{*}\left(S_{p}^{1 / 2} f^{(1)}\right)+b^{* t}\left(S_{p}^{1 / 2} f^{(2)}\right) .
\end{aligned}
$$

If we define $\bar{H}=\left(I-S_{0}\right)^{1 / 2}\left(I-S_{p}\right)^{1 / 2}+S_{0}^{1 / 2} S_{p}^{1 / 2}$ and $\bar{L}=S_{0}^{1 / 2}\left(I-S_{p}\right)^{1 / 2}-$ $\left(I-S_{0}\right)^{1 / 2} \mathrm{~S}_{p}^{1 / 2}, \bar{H}$ and $\bar{L}$ satisfy (3.3) and because $S_{p}-S_{0}$ is trace class, $\bar{L}$ is HilbertSchmidt (by hypothesis and Lemma 4.1 in [32], $S_{0}^{1 / 2}-S_{p}^{1 / 2}$ and $\left(I-S_{0}\right)^{1 / 2}-$ $\left(I-S_{p}\right)^{1 / 2}$ are Hilbert-Schmidt; but $\bar{L}=\left(S_{0}^{1 / 2}-S_{p}^{1 / 2}\right)\left(I-S_{p}\right)^{1 / 2}+\left(\left(I-S_{p}\right)^{1 / 2}-\right.$ $\left.\left(I-S_{0}\right)^{1 / 2}\right) S_{p}^{1 / 2}$ and thus is itself Hilbert-Schmidt). Therefore, there exists a unitary $W$ such that

$$
\begin{gathered}
\overline{\mathscr{A}}(f) \equiv W\left(a\left(f^{(1)}\right)+a^{t}\left(f^{(2)}\right)\right) W^{*}=a\left(\bar{H} f^{(1)}\right)+a^{t}\left(\bar{H} f^{(2)}\right)+b^{*}\left(\bar{L} f^{(1)}\right)+b^{* t}\left(\bar{L} f^{(2)}\right), \\
\overline{\mathscr{B}}^{*}(f) \equiv W\left(b^{*}\left(f^{(1)}\right)+b^{* t}\left(f^{(2)}\right)\right) W^{*}= \\
-a\left(\bar{L} f^{(1)}\right)-a^{t}\left(\bar{L} f^{(2)}\right)+b^{*}\left(\bar{H} f^{(1)}\right) \\
+b^{* t}\left(\bar{H} f^{(2)}\right) .
\end{gathered}
$$

But this implies

$$
\overline{\mathscr{A}}\left(\left(I-S_{p}\right)^{1 / 2} f\right)+\overline{\mathscr{B}}^{*}\left(S_{p}^{1 / 2} f\right)=\mathscr{A}_{0}(f)
$$


so that

$$
\mathscr{A}_{0}(f)=W \mathscr{A}_{p}(f) W^{*} .
$$

Since we have already established that the representations $\left\{\mathscr{A}_{0}(f), \mathscr{H}, \Omega_{0}\right\},\left\{\mathscr{A}_{p}(f)\right.$, $\left.\mathscr{H}, \Omega_{0}\right\}$ are unitarily equivalent to the representations of $\mathscr{E}(K)$ induced by $\phi_{0}^{\prime}, \phi_{p}^{\prime}$, respectively, the assertion (a) in the theorem is proven. The argument for (b) is clearly the same.

We shall now verify that the conditions (1) and (2) of the theorem do indeed obtain. In a previous version of this paper we proved condition (1) in two (and four) space-time dimensions by using the antilocality of certain operators [41] in a manner analogous to that in [8] (see Lemmas 3.1 and 3.4 in [8]). As, however, the antilocality of the relevant operators is only known in an odd number of space dimensions [41] and since the argument depended in any case on the details of the representation (that differ, as well, in 1, 2 or 3 space dimensions), we present a simpler proof depending only on the form of the quasifree states and the fact that the vacuum is cyclic and separating for the free Fermi algebra $\mathfrak{F}(\underline{\mathcal{O}})$, for $\underline{\mathcal{O}} \subset \mathbb{R}^{d-1}$ such that the interior of the complement of $\underline{O}$ in $\mathbb{R}^{d-1}$ is not empty [46]. This argument is, thus, independent of dimension $d$. The form of $S_{0}$ and $S_{p}$ for $d=4(d=3$ is similar $)$ is given later.

Lemma 3.3. 0 and 1 are not eigenvalues of $S_{0}$ and $S_{p}$ in $L_{2}(\underline{O})^{\oplus 2}\left(\right.$ for $d=3,4, S_{0}$ and $S_{p}$ are operators on $L_{2}(\underline{\mathcal{O}})^{\oplus d}$ ).

Proof. Assume there is an $F \in K_{0}(\underline{\mathcal{O}})$ such that $S_{0} F=0$. Then $\phi_{0}\left(B(F)^{*} B(F)\right)=0$, so that

$$
\left\langle\Omega_{0}, B(F)^{*} B(F) \Omega_{0}\right\rangle=\left\|B(F) \Omega_{0}\right\|^{2}=0,
$$

which implies that $B(F) \Omega_{0}=0$. But, as the vacuum is separating for $\mathfrak{F}(\underline{\mathcal{O}}), B(F)=0$. This is, however, not possible unless $F=0$, since $\left[B(F)^{*}, B(F)\right]_{+}=\|F\|^{2} I$. Thus, 0 cannot be an eigenvalue for $S_{0}$.

Let, now, $F \in K_{p}(\underline{\Theta})$ be such that $S_{p} F=0$. This is equivalent to $S_{p} F_{i}=$ $0, i=1,2\left(F=F_{1}+F_{2}\right.$ is the orthogonal decomposition of $\left.F, F_{i} \in K_{0}\left(\underline{\mathcal{Q}}_{i}\right)\right)$, since $S_{p} K_{0}\left(\underline{\mathcal{O}}_{i}\right) \subseteq K_{0}\left(\underline{\mathcal{O}}_{i}\right)$. This reduces to the previous case with $\underline{\mathcal{O}}$ chosen equal to $\underline{\mathcal{O}}_{i}$. Thus, again, $F=0$ and 0 is not an eigenvalue of $S_{p}$.

If there is an $F \in K_{0}(\underline{\mathcal{O}})$ such that $S_{0} F=F$, then

$$
\phi_{0}\left(B(F) B(F)^{*}\right)=-\phi_{0}\left(B(F)^{*} B(F)\right)+\langle F, F\rangle_{K_{0}(\mathcal{O})}=0 .
$$

Thus, as above, one obtains $\left\|B(F)^{*} \Omega_{0}\right\|^{2}=0$, yielding $B(F)^{*}=0$, as before. Thus, $F=0$ and 1 is not an eigenvalue of $S_{0}$. The proof that 1 is not an eigenvalue for $S_{p}$ is now clear.

We next verify condition (2) of Theorem 3.2.

Proposition 3.4. $S_{0}-S_{p}$ is a trace class operator on $L_{2}(\underline{\mathcal{O}})^{\oplus 2}$.

Proof. Writing

$$
E_{i}=\left(\begin{array}{cc}
E_{i}^{\prime} & 0 \\
0 & E_{\imath}^{\prime}
\end{array}\right), \quad i=1,2
$$


a simple calculation yields

$$
S_{0}-S_{p}=\frac{1}{2}\left(\begin{array}{cc}
E_{1}^{\prime} C_{-}^{2} E_{2}^{\prime} & E_{1}^{\prime} C_{-} C_{+} E_{2}^{\prime} \\
E_{1}^{\prime} C_{+} C_{-} E_{2}^{\prime} & E_{1}^{\prime} C_{+}^{2} E_{2}^{\prime}
\end{array}\right)+\frac{1}{2}\left(\begin{array}{cc}
E_{2}^{\prime} C_{-}^{2} E_{1}^{\prime} & E_{2}^{\prime} C_{-} C_{+} E_{1}^{\prime} \\
E_{2}^{\prime} C_{+} C_{-} E_{1}^{\prime} & E_{2}^{\prime} C_{+}^{2} E_{1}^{\prime}
\end{array}\right) .
$$

Thus, $S_{0}-S_{p}$ will be trace class (tc) on $L_{2}(\underline{\mathcal{O}})^{\oplus 2}$ if the operators $E_{i}^{\prime} C_{ \pm}^{2} E_{j}^{\prime}$ and $E_{i}^{\prime} C_{-} C_{+} E_{j}^{\prime}, i \neq j$, are trace class in $L_{2}(\mathbb{R})$.

We pause for definitions. Let $\Theta_{1}(x) \in \mathscr{S}(\mathbb{R})$ such that $\Theta_{1}(x)=1, x \in \underline{\mathcal{O}}_{1}, \Theta_{2}(x) \in$ $\mathcal{O}_{M}(\mathbb{R})$ (the space of slowly increasing $C^{\infty}$ functions) such that $\Theta_{2}(x)=1, x \in \underline{\mathcal{O}}_{2}$, and require further that $\operatorname{dist}\left(\operatorname{supp} \Theta_{1}\right.$, supp $\left.\Theta_{2}\right)>0$. Let $\Delta_{i}, i=1,2$, be the operator of multiplication by $\Theta_{i}(x)$. One has, clearly, $E_{i}^{\prime} \Delta_{i}=E_{i}^{\prime}=\Delta_{i} E_{i}^{\prime}, i=1,2$. Note that $C_{-} C_{+}=m_{f} / \omega$ and $C_{ \pm}^{2}=1 \pm i \nabla \omega^{-1}$.

The function

$$
\omega^{-1}(x)=(2 \pi)^{-1} \int d p\left(p^{2}+m_{f}^{2}\right)^{-1 / 2} e^{-i p x}
$$

is $C^{\infty}$ for $x \neq 0$, and it and all of its derivatives decrease faster than any inverse power of $|x|$ as $|x| \rightarrow \infty$ (this can be seen with a bit of contour integration). Thus, the kernel $\left(\Delta_{1} \omega^{-1} \Delta_{2}\right)(x, y)=\Theta_{1}(x) \omega^{-1}(\mathrm{x}-\mathrm{y}) \Theta_{2}(y)$ in configuration space is in $\mathscr{S}\left(\mathbb{R}^{2}\right)\left(\underline{Q}_{1}\right.$ is bounded!), so that the operator $\Delta_{1} \omega^{-1} \Delta_{2}$ is tc (see, e.g. [9, p. 266]). Therefore, for $i \neq j$,

$$
E_{i}^{\prime} C_{-} C_{+} E_{j}^{\prime}=E_{i}^{\prime} \Delta_{i} C_{-} C_{+} \Delta_{j} E_{j}^{\prime}
$$

is tc. Noting that $\Delta_{i} C_{ \pm}^{2} \Delta_{j}= \pm i \Delta_{i} \nabla \omega^{-1} \Delta_{j}$, for $i \neq j$ ( 1 is a local operator), the properties of the derivatives of $\omega^{-1}$ given above yield the conclusion that $E_{i}^{\prime} C_{ \pm}^{2} E_{j}^{\prime}$ is also tc for $i \neq j$.

This completes the proof of the existence of normal product states and the funnel property for the free Fermi algebra in two space-time dimensions. We next want to outline the proof for the four space-time dimensional, free, spin $1 / 2$ field. (The proof for three space-time dimensions is similar, but we give no details here.) As in [11] we define the bounded operators $S, T$, from $L_{2}\left(\mathbb{R}^{3}\right)^{\oplus 2}$ to $L_{2}\left(\mathbb{R}^{3}\right)$ by

$$
\begin{aligned}
& S F=\left(\frac{1+n_{3}}{2}\right)^{1 / 2} f^{(1)}-\frac{n_{1}+i n_{2}}{\left(2\left(1+n_{3}\right)\right)^{1 / 2}} f^{(2),} \\
& T F=\left(\frac{1-n_{3}}{2}\right)^{1 / 2} f^{(1)}+\frac{n_{1}+i n_{2}}{\left(2\left(1-n_{3}\right)\right)^{1 / 2}} f^{(2)},
\end{aligned}
$$

where $n_{l}=i\left(-\sum_{k} \partial^{2} / \partial x_{k}^{2}\right)^{-1 / 2} \partial / \partial x_{l}$. We define, further, the operators $A, B$, from $L_{2}\left(\mathbb{R}^{3}\right)^{\oplus 4}$ to $L_{2}\left(\mathbb{R}^{3}\right)^{\oplus 2}$ by $\left(\omega=\left(-\sum_{k} \partial^{2} / \partial x_{k}^{2}+m_{f}^{2}\right)^{1 / 2}\right)$

$$
A=\left(\begin{array}{lc}
\left(\frac{\omega+m_{f}}{2 \omega}\right)^{1 / 2} T & -\left(\frac{\omega-m_{f}}{2 \omega}\right)^{1 / 2} T \\
\left(\frac{\omega+m_{f}}{2 \omega}\right)^{1 / 2} S & \left(\frac{\omega-m_{f}}{2 \omega}\right)^{1 / 2} S
\end{array}\right),
$$


and

$$
B=\left(\begin{array}{lr}
\left(\frac{\omega-m_{f}}{2 \omega}\right)^{1 / 2} S & -\left(\frac{\omega+m_{f}}{2 \omega}\right)^{1 / 2} S \\
\left(\frac{\omega-m_{f}}{2 \omega}\right)^{1 / 2} T & \left(\frac{\omega+m_{f}}{2 \omega}\right)^{1 / 2} T
\end{array}\right) .
$$

The time zero field is given by [11]

$$
\Psi_{0}(F)=a(A F)+b^{*}(B F),
$$

where $F \in L_{2}\left(\mathbb{R}^{3}\right)^{\oplus 4}$ and $a, b$ provide two Fock representations over $L_{2}\left(\mathbb{R}^{3}\right)^{\oplus 2}$ on $\underline{\mathscr{F}}_{f}$ so that $[a(f), b(g)]_{+}=0$ for any $f, g \in L_{2}\left(\mathbb{R}^{3}\right)^{\oplus 2}$. The field $\Psi_{0}(F)$ satisfies $\left[\Psi_{0}(F)^{*}, \Psi_{0}(G)\right]_{+}=\langle F, G\rangle_{L_{2}\left(\mathbb{R}^{3}\right)^{\oplus 4}}$. The vacuum state $\phi_{0}(\cdot)$ is determined by its two-point function

$$
\phi_{0}\left(\Psi_{0}(F)^{*} \Psi_{0}(G)\right)=\left\langle F, B^{*} B G\right\rangle_{L_{2}\left(\mathbb{R}^{3}\right)^{\oplus 4}}
$$

so that, defining $K(\underline{O})=\mathscr{P}(\mathcal{Q})^{\oplus 4}, \mathcal{O} \subset \mathbb{R}^{3}$, and $K_{0}(\mathcal{O})$ as the completion of $K(\underline{O})$ in the $L_{2}\left(\mathbb{R}^{3}\right)^{\oplus 4}$ norm $\left(\|\cdot\|_{0}\right)$, i.e. $K_{0}(\underline{\mathcal{O}})=L_{2}(\underline{\mathcal{O}})^{\oplus 4}$, the counterpart of the operator $S_{0}$ in the two dimensional case is $E B^{*} B E$ (call it again $S_{0}$ ), where $E$ is the orthogonal projection in $L_{2}\left(\mathbb{R}^{3}\right)^{\oplus 4}$ onto $K_{0}(\mathcal{O})$. With the obvious parallel definitions, the state $\phi_{p}, \mathscr{A}\left(K_{p}(\underline{\mathcal{O}})\right)$ is determined by

$$
\phi_{p}\left(\Psi_{0}(F)^{*} \Psi_{0}(G)\right)=\left\langle F, S_{p} G\right\rangle_{p},
$$

with $S_{p}=E_{1} B^{*} B E_{1} \Theta_{1}+E_{2} B^{*} B E_{2} \Theta_{2}$. The states $\phi_{0}^{\prime}\left\lceil\mathscr{E}\left(K_{0}(\underline{\mathcal{O}})\right)\right.$ and $\phi_{p}^{\prime}\left\lceil\mathscr{E}\left(K_{p}(\underline{\mathcal{O}})\right)\right.$ are determined analogously. Due to $K_{0}(\underline{\mathcal{O}})=K_{p}(\underline{\mathcal{O}})$ (which is trivial), Theorem 3.2 will give us the existence of normal product states as before when we prove

Proposition 3.5. 1) $S_{0}$ and $S_{p}$ do not have eigenvalues 0 and 1 in $K_{0}(\underline{O})$.

2) $S_{0}-S_{p}$ is a trace class operator on $K_{0}(\underline{Q})$.

Proof. (1) follows, of course, from Lemma 3.3.

To prove (2) we note that since $E_{1}+E_{2}=E$,

$$
S_{0}-S_{p}=E_{1} B^{*} B E_{2}+E_{2} B^{*} B E_{1} .
$$

A simple calculation tells us that $S_{0}-S_{p}$ is tc if the operators

$$
E_{i}^{\prime} \frac{\omega \pm m_{f}}{2 \omega} E_{j}^{\prime} \text { and } E_{t}^{\prime} \frac{\sqrt{\omega^{2}-m_{f}^{2}}}{2 \omega} n_{3} E_{j}^{\prime}
$$

$i \neq j$, are tc on $L_{2}\left(\mathbb{R}^{3}\right)$, where $E_{i}^{\prime}$ is the projection in $L_{2}\left(\mathbb{R}^{3}\right)$ onto $L_{2}\left(\underline{O}_{i}\right)$. We note that

$$
\frac{\omega \pm m_{f}}{2 \omega}=\frac{1}{2}\left(1 \pm \frac{m_{f}}{\omega}\right) \text { and } \frac{\sqrt{\omega^{2}-m_{f}^{2}}}{2 \omega} n_{3}=i \frac{\partial}{\partial x_{3}}(2 \omega)^{-1}
$$

Moreover, defining the operators $\Delta_{i}, i=1,2$, in an analogous fashion to $\Delta_{1}, \Delta_{2}$ in the proof of Proposition 3.4, we have $E_{i}^{\prime} \Delta_{i}=E_{i}^{\prime}=\Delta_{i} E_{i}^{\prime}$. Since the properties of 
$\omega^{-1}(x)$ listed in the proof of Proposition 3.4 hold in three dimensions as well as one, the argument developed there may be employed to conclude

$$
\Delta_{i}\left(1 \pm \frac{m_{f}}{\omega}\right) \Delta_{j}= \pm m_{f} \Delta_{i} \omega^{-1} \Delta_{j}
$$

and $i \Delta_{i} \partial \omega^{-1} / \partial x_{3} \Delta_{j}$ are trace class whenever $i \neq j$, completing the proof of Proposition 3.5.

We would like to end this chapter with a final comment. If $\mathscr{F}$ is generated by a net of algebras $\{\mathfrak{F}(\mathcal{O})\}$, such that for every bdc $\mathcal{O}, \mathfrak{F}(\mathcal{O})=\mathfrak{A}(\mathcal{O}) \otimes \mathfrak{B}(\mathcal{O})$, then if the nets $\{\mathfrak{U}(\mathcal{O})\}$ and $\{\mathfrak{B}(\mathcal{O})\}$ satisfy individually the funnel property, $\mathscr{F}$ satisfies the funnel property. This can be immediately seen once one remarks that the direct product of two type $I$ factors is again a type $I$ factor [38, Propositions 2.6.2 and 2.6.7]. Thus, the net $\{\mathfrak{F}(\mathcal{O})\}$ given by the tensor product of the free Fermi field algebra and free Bose algebra satisfies the funnel property. We shall use this fact in the next chapter.

\section{Application to the Yukawa ${ }_{2}+\boldsymbol{P}(\phi)_{2}$ Quantum Field Model}

In this final chapter we demonstrate that the theory discussed in Chap. II can be applied to the quantum field model with the nonlinear scalar (or pseudoscalar) Yukawa interaction: $\bar{\Psi} \Gamma \Psi \phi:, \Gamma=1$ (or $i \gamma_{5}$ ), plus a boson selfinteraction: $P(\phi)$ : in two space-time dimensions $(P(\phi)$ is an arbitrary polynomial that is bounded from below). Since most of the conditions in Theorem 2.1 concern the reference (in our case also the defining) representation $\pi_{0}$, which we take to be the relativistic BoseFermi Fock representation, we shall first constate the necessary properties for the free Bose and Fermi fields in two space-time dimensions. (We remark that all the results stated for the free fields are valid in four space-time dimensions, as well). As most of the results concerning the free fields are well-known, we shall be rather sketchy.

We denote by $\mathscr{F}=\mathscr{F}_{b} \otimes \mathscr{F}_{f}$ the Fock space for a Bose particle of mass $m_{b}>0$ and fermions of mass $m_{f}>0$, where $\mathscr{F}_{b}, \mathscr{F}_{f}$, is the standard direct sum over the totally symmetrized, respectively anti-symmetrized, one-particle space $L_{2}\left(\mathbb{R}^{2}\right)$. It is well known (see, e.g. [17]) that the local von Neumann algebra $\mathfrak{F}(\mathcal{O})$ in $\mathscr{F}$, generated by the operators $\Psi(f)$ and $\exp \{i(\phi(f)+\Pi(g))\}$ with supp $f, g \subset \mathcal{O}$ and $\Pi(g)=$ $\phi(d g / d t)$, coincides with the time zero algebra $\mathfrak{F}(\underline{\mathcal{O}})$ generated by the same operators at $t=0$ and with suitably restricted test functions, if $\mathcal{O}$ is a bounded spacetime region containing the time zero base $\mathcal{O}$ of the double cone containing $\mathcal{O}$. Further, it is clear that $\mathfrak{F}(\mathcal{O})=\mathfrak{H}_{b}(\mathcal{O}) \otimes \mathfrak{F}_{f}(\mathcal{O})$, where $\mathfrak{A}_{b}(\mathcal{O})$ is generated by the $\exp \{i(\phi(f)+\Pi(g))\}$ and $\mathfrak{F}_{f}(\mathcal{O})$ by the $\Psi(f)$. As it is known that $\mathfrak{U}_{b}(\underline{\mathcal{O}})$ is a factor of type III $[2,11]$ and $\mathfrak{F}_{f}(\underline{\mathcal{O}})$ is a factor $[11], \mathfrak{A}_{b}(\mathcal{O}) \otimes \mathfrak{F}_{f}(\mathcal{O})$ is a type III factor $[38$, Theorem 2.6.4 and Prop. 2.6.7] and thus simple as a $C^{*}$-algebra, since Fock space is separable (countably decomposable type III factors contain no proper norm-closed two-sided ideals - follows from [38, Lemma 4.1.5]). $\mathscr{F}$ is therefore also simple [38, Prop. 1.23.8] (we point this out, although it is not necessary for the application of our theorem, for the benefit of readers of an earlier version of this paper). It is, in addition, weakly dense in $\mathfrak{B}(\mathscr{F})$. 
If $N$ denotes the fermion number operator on $\mathscr{F}, e^{i \theta N}, \theta \in[0,2 \pi]$, defines a strongly continuous unitary representation of the circle group $U(1)$, which we take as our gauge group $\mathscr{G}$. (Of course, $e^{i \theta N}=e^{i \theta Q}$, where $Q$ is the total charge operator.) This generates an automorphism group $\alpha_{g}, g \in \mathscr{G}$, on $\mathscr{F}$ by

$$
\alpha_{\theta}(F)=e^{i \theta N} F e^{-i \theta N} \text {. }
$$

The twist for the free Fermi field is given as in the preceding chapter by $\mathfrak{F}^{t}(\mathcal{O})=$ $Z \mathfrak{F}(\mathcal{O}) Z^{-1}$, where $Z=(1-i) / 2+(1+i) e^{i \pi N} / 2$.

As already mentioned, in four space-time dimensions duality for the free Bose algebra $\mathfrak{A}_{b}(\mathcal{O})[1]$ and twisted duality (with the twist defined above) for the free Fermi algebra $\mathfrak{F}_{f}(\mathcal{O})[11,12]$ are known, yielding twisted duality for $\mathfrak{F}(\mathcal{O})$ (using Tomita's commutant theorem, i.e. for $\mathfrak{A}=\mathfrak{B} \otimes \mathfrak{C}, \mathfrak{B}$ and $\mathfrak{C}$ von Neumann algebras, $\mathfrak{U}^{\prime}=\mathfrak{B}^{\prime} \otimes \mathfrak{C}^{\prime}$ ). Driessler sketched the proof of duality of the free Bose field in two space-time dimensions in [14], and the argument in [12] translating Dell'Antonio's structure results into twisted duality is dimension independent. In the appendix we outline the proof of the corresponding structure results for the two space-time dimensional fermions.

It is known [1] that $\mathfrak{U}_{b}\left(\mathcal{O}_{1}\right)^{\prime} \cap \mathfrak{U}_{b}\left(\mathcal{O}_{2}\right)^{\prime \prime}=\mathscr{A}_{b}\left(\mathcal{O}_{1}^{\prime}\right)^{\prime \prime} \cap \mathfrak{A}_{b}\left(\mathcal{O}_{2}\right)^{\prime \prime}=\mathfrak{A}_{b}\left(\mathcal{O}_{1}^{\prime} \cap \mathcal{O}_{2}\right)^{\prime \prime}$ for $\mathcal{O}_{1}, \mathcal{O}_{2}$ arbitrary open double cones with bases at a common time. Although this is not true for the free Fermi observable algebra (as we have defined it), we have:

Proposition 4.1. For arbitrary bounded double cones $\mathcal{O}_{1}, \mathcal{O}_{2} \subset \mathbb{R}^{2}$ with bases at a common time, $\mathscr{F}_{f}\left(\mathcal{O}_{1}^{\prime}\right)^{\prime \prime} \cap \mathfrak{F}_{f}\left(\mathcal{O}_{2}\right)=\mathfrak{F}_{f}\left(\mathcal{O}_{1}^{\prime} \cap \mathcal{O}_{2}\right)$.

Proof. The inclusion $\mathfrak{F}_{f}\left(\mathcal{O}_{1}^{\prime} \cap \mathcal{O}_{2}\right) \subseteq \mathscr{F}_{f}\left(\mathcal{O}_{1}^{\prime}\right)^{\prime \prime} \cap \mathfrak{F}_{f}\left(\mathrm{O}_{2}\right)$ is clear. Using twisted duality the opposite inclusion is equivalent to

$$
\left(\mathscr{F}_{f}^{t}\left(\mathcal{O}_{1}\right) \vee \mathscr{F}_{f}^{t}\left(\mathcal{O}_{2}^{\prime}\right)^{\prime \prime}\right)^{\prime \prime} \supset \mathscr{F}_{f}^{t}\left(\mathcal{O}_{1} \cup \mathcal{O}_{2}^{\prime}\right)^{\prime \prime}
$$

This is itself equivalent to

$$
\left(\mathscr{F}_{f}\left(\mathcal{O}_{1}\right) \vee \mathscr{F}_{f}\left(\mathcal{O}_{2}^{\prime}\right)^{\prime \prime}\right)^{\prime \prime} \supset \mathscr{F}_{f}\left(\mathcal{O}_{1} \cup \mathcal{O}_{2}^{\prime}\right)^{\prime \prime}
$$

since the twist is implemented by a unitary operator. Polynomials in the set $\left\{\Psi_{0}(f) \mid f \in K\left(\underline{\Theta}_{1} \cup \underline{\mathcal{O}}_{2}^{\prime}\right), \Psi_{0}\right.$ the time-zero Fermi field $\}$ (see previous chapter) are dense in $\mathscr{F}{ }_{f}\left(\mathcal{O}_{1} \cup \mathcal{O}_{2}^{\prime}\right)^{\prime \prime}$, and as $\underline{O}_{1}, \underline{O}_{2}^{\prime}$ are open, the existence of a partition of unity in $\mathscr{S}(\mathbb{R})$ over arbitrary open coverings of an arbitrary open set in $\mathbb{R}$ entails there are $f_{1} \in \mathscr{S}\left(\underline{\mathcal{O}}_{1}\right)$, $f_{2} \in \mathscr{S}\left(\underline{Q}_{2}^{\prime}\right)$, such that $f=f_{1}+f_{2}$. By the linearity of the operators $\Psi_{0}(f),(4.1)$ follows at once.

Remarks. This proposition is valid in four space-time dimensions, using the same argument.

Thus, for arbitrary bdc's $\mathcal{O}, \mathcal{O}_{1}$, we have, by twisted duality,

$$
\begin{aligned}
& \pi_{0}\left(\mathfrak{F}^{t}(\mathcal{O})^{c} \cap \mathfrak{F}\left(\mathcal{O}_{1}\right)\right) \subseteq \mathfrak{F}^{t}(\mathcal{O})^{\prime} \cap \mathfrak{F}\left(\mathcal{O}_{1}\right) \\
& =\mathscr{F}\left(\mathcal{O}^{\prime}\right)^{\prime \prime} \cap \mathfrak{F}\left(\mathcal{O}_{1}\right)=\mathscr{A}_{b}\left(\mathcal{O}^{\prime}\right)^{\prime \prime} \otimes \mathscr{F}_{f}\left(\mathcal{O}^{\prime}\right)^{\prime \prime} \cap \mathfrak{A}_{b}\left(\mathcal{O}_{1}\right) \otimes \mathfrak{F}_{f}\left(\mathcal{O}_{1}\right) \\
& =\mathfrak{U}_{b}\left(\mathcal{O}^{\prime} \cap \mathcal{O}_{1}\right) \otimes \mathfrak{F}_{f}\left(\mathcal{O}^{\prime} \cap \mathcal{O}_{1}\right)=\mathfrak{F}\left(\mathcal{U}^{\prime} \cap \mathcal{O}_{1}\right)
\end{aligned}
$$

( $\pi_{0}$ is the Fock representation), which verifies condition (2) in Theorem 2.1. 
Moreover, since $\pi_{0}\left(\mathfrak{F}^{t}(\mathcal{O})^{e} \cap \mathfrak{F}\left(\mathcal{O}_{1}\right)\right) \subset \mathbb{F}^{t}(\mathcal{O})^{\prime} \cap \mathfrak{F}\left(\mathcal{O}_{1}\right)$, condition $\left(2^{\prime}\right)$ in Theorem 2.8 is also satisfied.

With the funnel property already proven for the free algebras $\{\mathfrak{F}(\mathcal{O})\}$ in Chap. III, it remains only to verify the necessary properties of the $Y_{2}+P(\phi)_{2}$ model. The dynamics of the $Y_{2}$ model were first constructed by Glimm and Jaffe in $[19,21]$. Further results, including the $P(\phi)_{2}$ interaction, were obtained by Schrader $[39,40]$ using the methods of [20]. They proved the existence of a state $\omega_{\text {ren }}(\cdot)$ on $\mathscr{F}$ as a weak *-limit of finite volume interacting $Y_{2}+P(\phi)_{2}$ states $\omega_{g}(\cdot)$ (equal to $\langle\Omega(g), \Omega(g)\rangle$, where $\Omega(g)$ is a vector in Fock space annihilated by the spatially cutoff Hamiltonian $H(g)$, depending on the cutoff function $g$ ) as $g \rightarrow 1$, that is described partially in the following theorem.

Theorem $4.2[40]$. There exists a separable Hilbert space $\mathscr{H}_{\mathrm{ren}}, a^{*}$-isomorphism $\pi$ of $\mathscr{F}$, a strongly continuous unitary representation $W_{\text {ren }}$ of the space-time translation group and a vector $\Omega_{\mathrm{ren}} \in \mathscr{H}_{\mathrm{ren}}$, cyclic for $\pi(\mathscr{F})$, such that

$$
\begin{aligned}
& \omega_{\text {ren }}(A)=\left\langle\Omega_{\text {ren }}, \pi(A) \Omega_{\text {ren }}\right\rangle \\
& W_{\text {ren }}(a, \tau)^{-1} \pi(A) W_{\text {ren }}(a, \tau)=\pi\left(\sigma_{a . \tau}(A)\right) \\
& W_{\text {ren }}(a, \tau) \Omega_{\text {ren }}=\Omega_{\text {ren }} \text { for all }(a, \tau) \in \mathbb{R}^{2} .
\end{aligned}
$$

The infinitesimal generator $H$ of the time translations is nonnegative. Further, for every bounded open set $\underline{\mathcal{O}} \subset \mathbb{R}$, there exists a unitary operator $U_{\mathscr{O}}: \mathscr{H}_{\mathrm{ren}} \rightarrow \mathscr{F}$ such that

$$
\pi(A)=U_{\mathscr{O}}^{*} A U_{\mathscr{O}}, \quad A \in \mathfrak{F}(\underline{\mathcal{O}})
$$

i.e. $\omega_{\text {ren }}$ and its corresponding representation $\pi$ are locally Fock on $\mathscr{F}$ (thus, locally normal on the product of the CCR and CAR algebras).

Remark. $(a, \tau) \rightarrow \sigma_{a, \tau}$ denotes the unique $*$-homomorphism of the space-time translation group into the group of automorphisms of $\mathscr{F}$, which is given on $\mathfrak{F}(\underline{O})$ by

$$
\sigma_{\alpha . \tau}(A)=V(a)^{-1} e^{i H(g) \tau} A e^{-i H(g) \tau} V(a), \quad A \in \mathfrak{F}(\underline{O}),
$$

where $g \in C_{0, \mathbb{R}}^{\infty}(\mathbb{R})$ (or a characteristic function) satisfies $g(x)=1$ on $\underline{Q}_{|a|+|\tau|}=$ $\{x \in \mathbb{R} \mid$ dist $(x, \underline{\mathcal{O}}) \leqq|a|+|\tau|\} . V(a)$ is the unitary representation of the space translations on $\mathscr{F}$. We comment that the Lorentz boosts are also canonically realized by a strongly continuous automorphism group [27]. See [19,21,40,27] for further details.

It is easy to see that since the Fock space vacuum and the interaction in an arbitrary finite volume are invariant under the gauge group defined above, one has, in addition, a strongly continuous unitary representation $U_{\text {ren }}(\cdot)$ of the gauge group on $\mathscr{H}_{\mathrm{ren}}$, locally generated by $e^{i \theta N}$, using the local Fock property. The twist operator on $\mathscr{H}_{\text {ren }}$ is given by

$$
Z_{\mathrm{ren}}=\frac{1-i}{2}+\frac{1+i}{2} U_{\mathrm{ren}}(\pi)
$$

Therefore, it only remains to verify that the representation $\pi$ of $\mathscr{F}$ given in Theorem 4.2 is irreducible, i.e. that $\omega_{\text {ren }}$ is a pure state for $\mathscr{F}$. If the spectrum 
condition were known for the representation $\left(\mathscr{H}_{\text {ren }}, \pi, \Omega_{\text {ren }}\right)$ and if $U_{\text {ren }}(\mathscr{G}) \subset 3_{\pi}^{\prime}$, where $3_{\pi}=\mathscr{F}_{\pi}^{\prime \prime} \cap \mathscr{F}_{\pi}^{\prime}$ is the center of $\mathscr{F}_{\pi}^{\prime \prime}$, one could perform a central decomposition of the state $\omega_{\text {ren }}$ with respect to the spectrum of $3_{\pi}$ as in [3] to obtain a set of representations $\left(\mathscr{H}_{\text {ren, } \zeta}, \pi_{\zeta}, \Omega_{\text {ren, } \zeta}\right)$ and a measure $\mu$ on 3 's spectrum so that

$$
\mathscr{H}_{\mathrm{ren}}=\int_{\operatorname{spec}(3)}^{\oplus} \mathscr{H}_{\mathrm{ren}, \zeta} d \mu(\zeta),
$$

etc., such that all the above properties are maintained and $\pi_{\zeta}(\mathscr{F})$ is irreducible in $\mathscr{H}_{\text {ren, } \zeta}$ for almost all $\zeta$. (The local Fock property for $\mathscr{H}_{\text {ren, } \zeta}$ follows again since each $\pi_{\zeta}$ will be a $*$-isomorphism between separable type III algebras $\mathfrak{F}(\underline{\mathcal{O}})$ and $\pi_{\zeta}(\mathfrak{F}(\mathcal{O}))$ and will be, thus, unitarily implementable). One could then apply Theorem 2.1 to the irreducible, locally normal, gauge representations $\left(\mathscr{H}_{\text {ren }, \zeta}, \pi_{\zeta}, \Omega_{\text {ren }, \zeta}\right)$ of $\left(\mathscr{F}, \mathscr{G}, \alpha, \mathscr{F}^{t}\right)$. Since the spectrum condition should hold in any reasonable quantum field model, only the condition $U(\mathscr{G}) \subset 3_{\pi}^{\prime}$ could be expected to fail, i.e. there could be non-gaugeinvariant "field objects at infinity". However, as pointed out in the remark made at the end of Chap. II, if the Haag-Kastler-Araki axioms hold in the representation $\left(\mathscr{H}_{\text {ren }}, \pi, \Omega_{\text {ren }}\right), \alpha_{g_{0}}(\cdot)$ will be unitarily implementable in (almost) all representations $\left(\mathscr{H}_{\text {ren }, \zeta}, \pi_{\zeta}, \Omega_{\text {ren, } \zeta}\right)$, which suffices for the application of our theorems.

Since, unfortunately, the spectrum condition has never been proven for the "Hamiltonian" construction of the $Y_{2}+P(\phi)_{2}$ model indicated above (but see [52] for the proof with periodic boundary conditions), much less the property $U(\mathscr{G}) \subset 3^{\prime}$, we shall take recourse to a construction through the Osterwalder-Schrader reconstruction theorem of a $Y_{2}\left(+P(\phi)_{2}\right)$ model satisfying the Wightman axioms using results of $[10,26]$ obtained by Euclidean methods. It will be necessary to establish the equivalence of the two constructions since we need the Wightman axioms from the one and the local Fock property from the other.

The space-time cutoff Euclidean Green's functions (EGF's) for $n$ bosons and $m$ fermion-antifermion pairs (by gauge invariance, if the number of fermions is not equal to the number of antifermions, the expectation is zero) are given through the Matthews-Salam-Seiler formula [42] by

$$
\begin{aligned}
& S_{\Lambda(L, T)}\left(x_{1}, \ldots, x_{n}, y_{1}, \ldots, y_{m}, z_{1}, \ldots, z_{m}\right) \\
& =Z(\Lambda)^{-1} \int_{\mathscr{S}^{\prime}\left(\mathbb{R}^{2}\right)} d \mu_{0}(\phi) \exp \left\{-\lambda \int_{\Lambda}: P(\phi):(x) d^{2} x\right\} \operatorname{det} S_{F}^{\prime}\left(y_{l}, z_{k} ; \phi_{\Lambda}\right) \times \\
& \quad \times \prod_{l=1}^{n} \phi\left(x_{l}\right) \operatorname{det}_{\mathrm{ren}}\left(1-\lambda K\left(\phi_{\Lambda}\right)\right),
\end{aligned}
$$

where

$$
Z(\Lambda)=\int d \mu_{0}(\phi) \exp \left\{-\lambda \int_{\Lambda}: P(\phi):(x) d^{2} x\right\} \operatorname{det}_{\mathrm{ren}}\left(1-\lambda K\left(\phi_{\Lambda}\right)\right),
$$

$d \mu_{0}(\phi)$ denotes the free Gaussian measure on $\mathscr{S}^{\prime}\left(\mathbb{R}^{2}\right)$ with mean zero and covariance $\left(-\Delta+m_{b}^{2}\right)^{-1}, \Lambda(=[-T / 2, T / 2] \times[-L / 2, L / 2])$ is a bounded rectangle in $\mathbb{R}^{2}$. $S_{F}^{\prime}\left(y_{i}, z_{k} ; \phi_{\Lambda}\right)$ is the kernel of

$$
S_{F}^{\prime}\left(f_{i}, g_{k} ; \phi_{A}\right)=\left\langle f_{i},(1-\lambda K)^{-1} S_{o} g_{k}\right\rangle_{L_{2}\left(\mathbb{R}^{2}\right)^{\oplus 2}} ;
$$

$\lambda$ is the Yukawa coupling constant (that we have, for simplicity's sake, set equal to 
the $P(\phi)_{2}$ coupling constant); $S_{o}$ is the free two point Schwinger function for the fermions:

$$
S_{o}(x-y)=\frac{1}{(2 \pi)^{2}} \int \frac{\not p+m_{f}}{p^{2}+m_{f}^{2}} e^{i p \cdot(x-y)} d^{2} p,
$$

where $\not p=p_{o} \gamma_{o}+p_{1} \gamma_{1}\left(\gamma_{i}\right.$ are the Euclidean $\gamma$-matrices (see [42])). $K=K\left(\phi_{A}\right)$ is the operator with kernel $K(x, y)=S_{o}(x-y) \phi(y) \chi_{\Lambda}(y)$, where $\chi_{A}$ is the characteristic function of $\Lambda$. The determinant in (4.3) is the $m \times m$ determinant of the matrix with elements $S_{F}^{\prime}\left(y_{i}, z_{k} ; \phi_{\Lambda}\right)$.

$$
\operatorname{det}_{\text {ren }}\left(1-\lambda K\left(\phi_{\Lambda}\right)\right)=\operatorname{det}_{3}\left(1-\lambda K\left(\phi_{A}\right)\right) \exp \left(-\lambda^{2} B_{A}\right),
$$

where

$$
\operatorname{det}_{3}(1-A)=\exp \left[\operatorname{Tr}\left(\ln (1-A)+A+A^{2} / 2\right)\right]
$$

and $B_{A}=\frac{1}{2}: \operatorname{Tr}\left(K^{2}+K^{*} K\right)$ : where $K^{*}$ is the adjoint of $K$ as an operator on $L_{2}\left(\mathbb{R}^{2}\right) \oplus L_{2}\left(\mathbb{R}^{2}\right)$. : : denotes Wick ordering with respect to $d \mu_{0}$. This description is somewhat formal, but Seiler demonstrated that by introducing momentum cutoffs the components of (4.3) are all well-defined as indicated and by performing the renormalization cancellations and removing the ultraviolet cutoff, the resulting integrand is indeed an integrable function with respect to the measure $d \mu_{0}(\phi)[42]$.

Independently, then, Cooper and Rosen [10] and Magnen and Sénéor [26] proved

Theorem 4.3. If $\lambda>0$ is sufficiently small, then:

1) the infinite volume EGF's $S(x ; y ; z)=\lim _{L, T \rightarrow \infty} S_{\Lambda}(x ; y ; z)$ exist as functionals in $\mathscr{S}^{\prime}\left(\mathbb{R}^{2(n+2 m)}\right)$; the limit is independent of the order taken.

2) the $S(x ; y ; z)$ satisfy all the Osterwalder-Schrader axioms [31], including exponential clustering. Hence the unique corresponding relativistic theory satisfies the Wightman axioms, including a mass gap.

Remarks. The original proofs of [10] and [26] were for the pure $Y_{2}$ model and with the assumption that $\lambda / m_{b}$ and $\lambda / m_{f}$ are sufficiently small. However, with estimates of [22] and arguments of [23] the above version of the original results can be proven.

The aim of the rest of this chapter is to prove that the two theories obtained in Theorems 4.2 and 4.3 are the same in the weak coupling limit. We begin by connecting space-time-cutoff objects in the Euclidean and Hamiltonian formalisms through a Feyman-Kac formula. One version of this formula was proven in [30] for the $Y_{2}+P(\phi)_{2}$ theory with ultraviolet cutoffs and no counterterms. A proof of the Feynman-Kac formula with cutoffs and counterterms was given in [43] (see particularly Theorems 3.2 and 3.3), and using the strong resolvent convergence of the Hamiltonian as one removes the ultraviolet cutoff [19] (see also [43]) and the results of [42] already mentioned, one obtains for $t_{i}>0, i=0, \ldots, n$,

$$
\begin{gathered}
\left\langle\Omega_{0}, e^{-t_{0} \hat{H}(L)} \prod_{j=1}^{n}\left\{\Psi^{\#}\left(\underline{x}_{j}, 0\right) e^{-t_{j} \hat{H}(L)}\right\} \Omega_{0}\right\rangle \\
=(Z S)_{\Lambda\left(L, \Sigma t_{j}\right)}\left(\left\{x_{j}, S_{j}\right\}_{j=1}^{n}\right),
\end{gathered}
$$


where the righthand side is the (unnormalized) cutoff EGF given in (4.3) with the appropriate number of bosons and fermion-antifermion pairs and with interactions in the space-time region $\Lambda=\left[s_{0}, s_{n+1}\right] \times[-L / 2, L / 2]$, where $s_{0}$ is arbitrary and $s_{j}=s_{j-1}+t_{j-1}, j=1, \ldots, n+1$. In the lefthand side (a vacuum expectation value in the relativistic Fock space $\mathscr{F}), \Psi^{\#}(\underline{x}, 0)$ is a time zero field $\left(\Psi^{\#}\right.$ denoting either $\Psi, \bar{\Psi}$ or $\phi$, where $\left.\bar{\Psi}=-i \Psi^{*} \gamma_{0}\right)$, and $\hat{H}(L)$ is the interacting Hamilton operator with space cutoff in the interval $[-L / 2, L / 2]$ with the conventional mass renormalization and Wick ordering, but with the energy counterterm of [43]. This Hamiltonian differs from the usual one [19] by a finite renormalization; thus, letting $E(L)$ denote the eigenvalue at the bottom of the spectrum of $\hat{H}(L)$, and defining $H(L)=\hat{H}(L)-E(L)$, $H(L)$ equals the Hamiltonian $H(g)$ in Theorem 4.2 with $g=\chi_{[-L / 2, L / 2]}[43$, Corollary 2.3]. Of course, Theorem 4.2 is still valid for dynamics given with this choice of renormalization constants. We adopt the normalization convention of [43], since the proof of the vacuum overlap (which we shall use below) in [43] uses Nelson's symmetry, which fails for the ordinary choice of vacuum renormalization counterterm (see, however, [49] for an alternative proof where the difference between counterterms is carried along).

If we denote by $\Omega(L)$ (a spatially cutoff interacting vacuum) a unit vector in $\mathscr{F}$ such that $H(L) \Omega(L)=0$, we know from [19] that there is a $\lambda_{0}(L)>0$ so that for all $\lambda_{0}(L) \geqq \lambda \geqq 0, \Omega(L)$ is unique. However, it may be that $\lambda_{0}(L) \downarrow 0$ as $L \rightarrow \infty$. Thus, we let $P(L)$ signify the projection in $\mathscr{F}$ onto the subspace (it is of finite dimension-see Theorem 5.2 in [19]) of vectors annihilated by $H(L)$. From the functional calculus for self-adjoint operators, one has (since $\operatorname{spec}(H(L) \subseteq[0, \infty)$ )

$$
s-\lim _{t \rightarrow \infty} e^{-t H(L)}=P(L),
$$

and because $P(L) \Omega_{0} \neq 0[43,49]$, one has

$$
\lim _{t \rightarrow \infty} e^{-t H(L)} \Omega_{0}=P(L) \Omega_{0} \neq 0
$$

Let $\Xi(L)=P(L) \Omega_{0} /\left\|P(L) \Omega_{0}\right\|$. A straightforward argument using (4.4) and (4.5) yields the space-time cutoff Gell-Mann-Low formula: for $t_{1}<t_{2}<\ldots<t_{n}$,

$$
\begin{aligned}
& \left\langle\Xi(L), \prod_{j=1}^{n-1}\left\{\Psi^{\#}\left(\underline{x}_{j}, 0\right) e^{-\left(t_{j+1}-t_{j}\right) H(L)}\right\} \Psi^{\#}\left(\underline{x}_{n}, 0\right) \Xi(L)\right\rangle \\
& \left.\left.=\lim _{T \rightarrow \infty} S_{\Lambda(L, T)}\left(\left\{\underline{x}_{j}, t_{j}\right)\right\}_{j=1}^{n}\right) \equiv S_{L}\left(\left\{\underline{x}_{j}, t_{j}\right)\right\}_{j=1}^{n}\right) .
\end{aligned}
$$

The existence of the righthand side is assured by Theorem 4.3, that of the lefthand side by (4.5). Recalling that the interacting fields (with space cutoff) are given by

$$
\Psi_{L}^{\#}(\underline{x}, t)=e^{i t H(L)} \Psi^{\#}(\underline{x}, 0) e^{-i t H(L)},
$$

we may conclude:

Theorem 4.4. The $S_{L}\left(\left\{\underline{x}_{j}, t_{j}\right)\right\}_{j=1}^{n}$ are the Schwinger functions (analytic continuation 
to imaginary time) of the spatially cutoff Wightman distributions

$$
\left\langle\Xi(L), \prod_{j=1}^{n} \Psi_{L}^{\#}\left(\underline{x}_{j}, t_{j}\right) \Xi(L)\right\rangle .
$$

Proof. Fix $L$ and $\lambda$ ( $\lambda$ in the range determined by Theorem 4.3). Because $H(L)$ is selfadjoint and positive and $\Xi(L)$ is invariant under $e^{i t H(L)}$, the expression (4.6) is a holomorphic function in $\left\{\left(t_{1}, \ldots, t_{n}\right) \in \mathbb{C}^{n} \mid \operatorname{Re} t_{1}<\operatorname{Re} t_{2}<\ldots<\operatorname{Re} t_{n}\right\}$ and the cutoff Wightman distributions are obtained as boundary values of this holomorphic function as $\operatorname{Re}\left(t_{i+1}-t_{i}\right) \downarrow 0, i=1, \ldots, n$.

In [43] it was proven that the following estimate holds in $Y_{2}+P(\phi)_{2}$ (for arbitrary but fixed coupling constant):

$$
\begin{gathered}
\left|S_{\Lambda(L, T)}\left(f_{1}, \ldots, f_{n} ; g_{1}, \ldots, g_{m} ; h_{1}, \ldots, h_{m}\right)\right| \\
\leqq(n !)^{1 / 2} C(L, T) \prod_{i, j}\left\|f_{i}\right\| g_{j}\left\|h_{j}\right\|
\end{gathered}
$$

for $\|$.$\| appropriate Schwartz space norms and C(L, T)$ a constant satisfying $\lim _{L, T \rightarrow \infty} C(L, T)<\infty$. Thus, using an argument of [28] that employs the Osterwalder-Schrader positivity in the time direction of the cutoff Schwinger functions (and not Euclidean invariance as in [31]), one obtains a uniform bound that, with the convergence of the Schwinger functions given by Theorem 4.3, entails that the boundary values - the spatially cutoff Wightman functions - converge in $\mathscr{S}^{\prime}$. (An alternative argument would be to use the $\phi$-bound below ((4.10a)) and the argument of [48] to yield the convergence as tempered distributions of the cutoff Wightman functions-the proof in [48] can be slightly modified to handle the fact that the $\phi$-bound in Theorem 4.5 has a Schwartz space norm in lieu of an $L_{1}$-norm). That is,

$$
\begin{aligned}
& W_{n+2 m}\left(x_{1}, \ldots, x_{n} ; y_{1}, \ldots, y_{m} ; z_{1}, \ldots, z_{m}\right) \\
& \quad=\lim _{L \rightarrow \infty}\left\langle\Xi(L), \prod_{j=1}^{n} \phi_{L}\left(\underline{x}_{j}, t_{j}\right) \prod_{j=1}^{m} \bar{\Psi}_{L}\left(\underline{y}_{l}, t_{i}\right) \prod_{k=1}^{m} \Psi_{L}\left(\underline{z}_{k}, t_{k}\right) \Xi(L)\right\rangle
\end{aligned}
$$

exists in $\mathscr{S}^{\prime}\left(\mathbb{R}^{2 n+4 m}\right)$ for small enough coupling constant. Further, by Theorems 4.3 and 4.4 , the Osterwalder-Schrader reconstruction theorem and the resultant unitary equivalence of the Wightman theories, the Wightman functions in (4.9) satisfy all the Wightman axioms including a positive mass gap. Since at every step the states are invariant under the gauge transformations, the gauge group is realized by a strongly continuous unitary representation $U_{W}(\mathscr{G})$ in the resultant Hilbert space $\mathscr{H}_{W}$. The unique vacuum, designated by $\Omega_{W}$, is invariant under $U_{W}(\mathscr{G})$.

The estimates of $[40]$ can be immediately applied to yield the fact that the state

$$
\omega_{\text {ren }}(A) \equiv \lim _{L \rightarrow \infty}\langle\Xi(L), A \Xi(L)\rangle, \quad \forall A \in \mathscr{F},
$$

exists and satisfies all the properties listed in Theorem 4.2. Because the vacuum expectation values of the two theories, $\left(\mathscr{H}_{W}, \Omega_{W}, \ldots\right)$ and $\left(\mathscr{H}_{\text {ren }}, \Omega_{\text {ren }}, \ldots\right)$ coincide, the uniqueness of the Wightman reconstruction theorem entails that $\mathscr{H}_{W}$ can be 
identified as a subspace of $\mathscr{H}_{\text {ren }}, \Omega_{W}$ with $\Omega_{\text {ren }}$, and the field operators on $\mathscr{H}_{W}$ with those on $\mathscr{H}_{\text {ren }}$. It is now necessary to explicitly call upon the $\phi$-bound $[48,43,49]$ :

Theorem 4.5. For arbitrary $f \in \mathscr{S}_{\mathbb{R}}\left(\mathbb{R}^{2}\right)$, we have in the sense of quadratic forms on $\mathscr{F}$,

$$
\pm \phi_{L}(f) \leqq\|f\|(H(L)+1)
$$

where $\|\cdot\|$ is a Schwartz space norm independent of $L$. Thus [48], in the sense of quadratic forms on $D\left(H^{1 / 2}\right) \times D\left(H^{1 / 2}\right) \subset \mathscr{H}_{\text {ren }} \times \mathscr{H}_{\text {ren }}$,

$$
\pm \phi_{W}(f) \leqq\|f\|(H+1),
$$

where $H$ is the generator of the time translations on $\mathscr{H}_{\text {ren }}$.

With the use of this $\phi$-bound a straightforward argument (see Proposition 1.1 in [48]) yields the equality of $\mathscr{H}_{W}$ and $\mathscr{H}_{\text {ren }}$ (note that $\left\|\Psi_{L}(f)\right\|_{\mathscr{F}} \leqq 1$, for every $L$, carries over to the infinite volume limit: $\left\|\Psi_{W}(f)\right\|_{\mathscr{H}_{\text {ren }}} \leqq 1$ ). Furthermore, utilizing the local Fock property, one sees that for all $f \in \mathscr{D}\left\langle\left(\mathbb{P}^{2}\right.\right.$ ) (the set of infinitely differentiable functions on $\mathbb{R}^{2}$ of compact support $), \pi\left(e^{i \phi(f)}\right)=\exp \left\{i \phi_{W}(f)\right\}$ on $\mathscr{H}_{\text {ren }}$. And since there is a partition of unity $\left\{\xi_{\alpha}\right\} \subset \mathscr{D}\left(\mathbb{R}^{2}\right)$, this holds for all $f \in \mathscr{Y}\left(\mathbb{R}^{2}\right)$. Thus it follows that the action of the unitary realizations $W_{W}(\cdot), W_{\mathrm{ren}}(\cdot), U_{W}(\cdot), U_{\mathrm{ren}}(\cdot)$, of the gauge and translation groups coincide on $\mathscr{H}_{\text {ren }}$, and that $U_{W}\left(P_{+}^{\uparrow}\right)$ implements the automorphism group $\left\{\sigma_{(\Lambda, a, \tau)}(\cdot) \mid(\Lambda, a, \tau) \in P_{+}^{\uparrow}\right\}$ of Theorem ${ }_{1}$ 4.2. Therefore we may conclude the following theorem.

Theorem 4.6. For small enough coupling constant $\lambda>0$ in the $\lambda Y_{2}+\lambda P(\phi)_{2}$ model, all of the limiting methods described above provide a locally Fock, gauge representation $(\mathscr{H}, \pi(\cdot), \Omega)$ of $\left(\mathscr{F}, \mathscr{G}, \alpha_{g}, \mathscr{F} t\right)$ with a cyclic vector $\Omega$ that is the unique vector invariant under the action of the Lorentz group, which is itself implemented by a strongly continuous unitary representation. Furthermore, the spectrum condition is satisfied.

Therefore, by a theorem of Araki [3], ( $\mathscr{H}, \pi(),. \Omega)$ provides, as well, an irreducible representation of $\mathscr{F}$, so that all the assumptions of Theorem 2.1 are fulfilled. We may thus decompose $(\mathscr{H}, \pi(\cdot), \Omega)$ with respect to the spectrum of $U(\mathscr{G})$ and reduce the representation of the observable algebra on the resultant (Abelian) coherent charge sectors. The observable algebras satisfy duality on all these charge sectors, and by Theorem $2.3 \pi(\mathscr{F})$ satisfies twisted duality. (Or one may apply Theorem 2.8 directly.) Thus, all assumptions in the axiomatic study of DHR [12] have been verified (weak additivity follows from the Wightman axioms) for this model.

Without going into details, we mention that by using ideas in [5] to introduce appropriate boundary conditions at infinity in the Hamiltonian approach and capitalizing upon the existence of two pure phases, i.e. two Wightman theories with unique vacua (selected by these boundary conditions), in the pseudoscalar $Y_{2}$ model in the large coupling (low temperature) limit-proven by Euclidean methods in [4] - , one may apply the arguments presented above to obtain, in this limit two ilngr's $\pi_{ \pm}$of $(\mathscr{F}, \mathscr{G}, \alpha, \mathscr{F})$ to which one can apply the theory of Chap. II. 


\section{Appendix: Structure Results on the Relativistic Free Fermi Field Algebra} for $d=2$

Defining operators $A$ and $B$ from $L_{2}(\mathbb{R})^{\oplus 2}$ to $L_{2}(\mathbb{R})$ by

$$
\begin{aligned}
& A F=2^{-1 / 2}\left\{C_{-} f^{(1)}-C_{+} f^{(2)}\right\}, \\
& B F=2^{-1 / 2}\left\{C_{+} f^{(1)}+C_{-} f^{(2)}\right\},
\end{aligned}
$$

where $F=\left\{f^{(1)}, f^{(2)}\right\}$ and $C_{ \pm}$are the operators defined in Chap. III, the time zero Fermi field can be written as

$$
\Psi_{0}(F)=a(A F)+b_{0}^{*}(B F)
$$

where $a, b_{0}$ give two Fock representations over $L_{2}(\mathbb{R})$ of the anticommutation relations on Fock space $\mathscr{F}_{f}$ and $\left[a(f), b_{0}(g)\right]_{+}=\left[a(f), b_{0}^{*}(g)\right]_{+}=0, \forall f, g \in L_{2}(\mathbb{R})$. In fact, referring back to Chap. III,

$$
\begin{gathered}
a(g)=\int b(k) \tilde{g}(k) d k, \\
b_{0}^{*}(g)=\int b^{*}(-k) \tilde{g}(k) d k,
\end{gathered}
$$

defines $a(g), b(g), \forall g \in L_{2}(\mathbb{R})$.

As in [11] we define an unbounded operator $\phi$ from a dense subset of $L_{2}(\underline{\mathcal{O}})^{\oplus 2}$ to a dense subset of $L_{2}\left(\underline{O}^{\prime}\right)^{\oplus 2}$ by

$$
A F=A \phi F, \quad F \in L_{2}(\underline{\mathcal{O}})^{\oplus 2} .
$$

To verify that $\phi$ is indeed defined as asserted, one first notes that $A \cdot G=0, G \in$ $L_{2}\left(\underline{Q}^{\prime}\right)^{\oplus 2}$, implies $G=0$. This follows because $A \cdot G=0$ entails $C_{-} g^{(1)}-C_{+} g^{(2)}=0$, so that $g^{(1)}=C_{-}^{-1} C_{+} g^{(2)}=(1 / m)(\omega+i \nabla) g^{(2)}$. But $\omega+i \nabla$ antilocal [41] implies $g^{(2)}$ $=g^{(1)}=0$. Thus, if there exists a $G \in L_{2}\left(\underline{\mathcal{O}}^{\prime}\right)^{\oplus 2}$ such that $A F=A G$, there is only one. Using again the antilocality of $\omega \pm i \nabla$, it is easy to show that $C_{-}^{-1} C_{+} \mathscr{S}\left(\underline{Q}^{\prime}\right)$ and $C_{+}^{-1} C_{-} \mathscr{S}\left(\underline{\mathcal{O}}^{\prime}\right)$ are dense in $L_{2}(\underline{\mathcal{O}})$, so for a dense set of $f \in L_{2}(\underline{\mathcal{O}})$, one can find a solution to the equation

$$
C_{-} f=C_{-} \xi-C_{+} \eta, \quad f \in L_{2}(\underline{\mathcal{O}}), \quad \xi, \eta \in L_{2}\left(\underline{\mathcal{O}}^{c}\right),
$$

and for another dense set there is a solution to

$$
C_{+} f=C_{-} \xi-C_{+} \eta \text {. }
$$

Thus, (A.1) admits a unique solution for a dense subset of $L_{2}(\underline{\mathcal{O}})^{\oplus 2}$. From the uniqueness of $\phi$ and the boundedness of $A$, one can conclude that $\phi$ is a closed operator. Further, the uniqueness in (A.1) implies that $\phi^{-1}$ exists and has a dense domain. Thus, one has the polar decomposition $U\left(\phi^{*} \phi\right)^{1 / 2}$, where $U$ is a unitary from $L_{2}(\underline{\mathcal{O}})^{\oplus 2}$ to $L_{2}\left(\underline{\mathcal{Q}}^{\prime}\right)^{\oplus 2}$. Moreover, because $A^{*} A+B^{*} B=1$, we may prove, following Dell'Antonio, that

$$
B G=-B \phi^{*} G, \quad G \in L_{2}\left(\underline{\mathcal{O}}^{c}\right)^{\oplus 2} \bigcap D\left(\phi^{*}\right),
$$

where $D\left(\phi^{*}\right)$ denotes the (dense) domain of $\phi^{*}$.

Therefore, all of the ingredients necessary to the analysis in Sect. 11 in [11] are verified, so that one can conclude the following structure properties of the local algebras $\mathfrak{F}_{f}(\underline{\mathcal{O}})$. 
Lemma A.1. 1) The Fock space $\mathscr{F}_{f}$ of the relativistic free Fermi field in two spacetime dimensions can be represented as an incomplete infinite tensor product with respect to a vector $\psi=\underset{i}{\otimes} \psi_{i}, \psi_{i} \in \mathscr{H}_{i} \otimes \mathscr{H}_{i}^{\prime}, \mathscr{F}_{f}=\underset{i}{\otimes}\left(\mathscr{H}_{i} \otimes \mathscr{H}_{i}^{\prime}\right)$, where $\mathscr{H}_{i}$ and $\mathscr{H}_{i}^{\prime}$ are two-dimensional Hilbert spaces. (This decomposition depends on the choice of $(\hat{O})$.

2) $\mathfrak{F}(\underline{O})=\left\{I \otimes \ldots \otimes\left(\mathfrak{B}\left(\mathscr{H}_{i}\right) \otimes I_{i}^{\prime}\right) \otimes \ldots \mid i=1,2, \ldots\right\}^{\prime \prime}$ $\mathscr{F}^{\prime}\left(\mathcal{O}^{\prime}\right)^{\prime \prime}=\left\{I \otimes \ldots \otimes\left(I_{i} \otimes \mathfrak{B}\left(\mathscr{H}_{i}^{\prime}\right)\right) \otimes \ldots \mid i=1,2, \ldots\right\}^{\prime \prime}$,

where the I's are the appropriate identity operators, so that $\mathfrak{F}(\underline{\mathcal{O}})=\mathscr{F}^{t}\left(\underline{\mathcal{O}}^{\prime}\right)^{\prime}$

3) $\mathfrak{F}(\underline{O})$ is a hyperfinite factor and is of type I if $\phi^{*} \phi\left(\phi^{*} \phi+1\right)^{-2}$ is trace-class and. type III otherwise.

Proof. The proof follows [11] and the appendix of [12], using (A.1) and (A.2) and the fact that $\phi$ has a polar decomposition with $U$ unitary.

Acknowledgements. The author wishes to thank Profs. John E. Roberts and Wulf Driessler for interesting discussions related to aspects of this work. Particular thanks is due to Professor Roberts for valuable criticism of an earlier version of this paper.

\section{References}

1. Araki, H.: von Neumann algebras of tocal observables for free scalar field. J. Math. Phys. 5, 1-13 (1964)

2. Araki, H.: Types of von Neumann algebras associated with free fields. Prog. Theor. Phys. 32, 956-965 (1964)

3. Araki, H.: On the algebra of all local observables. Prog. Theor. Phys. 32, 844-854 (1964)

4. Balaban, T., Gawedzki, K.: A Low temperature expansion for the pseudoscalar Yukawa model of quantum fields in two space-time dimensions. Ann. Inst. H. Poincaré.

5. Béllissard, J., Fröhlich, J., Gidas. B.: Soliton mass and surface tension in the $\left(\lambda|\phi|{ }^{4}\right)_{2}$ quantum field model. Commun. Math. Phys. 60, 37-72 (1978)

6. Bisognano, J. J., Wichmann, E. H.: On the duality condition for a hermitian scalar field. J. Math. Phys. 16, 985-1007 (1975)

7. Bisognano, J. J., Wichmann, E. H.: On the duality condition for quantum fields. J. Math. Phys. 17, 303-321 (1976)

8. Buchholz, D.: Product states for local algebras. Commun. Math. Phys. 36, 287--304 (1974)

9. Cochran, J. A.: The analysis of linear integral equations. New York: McGraw-Hill 1972

10. Cooper, A., Rosen, L.: The weakly coupled Yukawa ${ }_{2}$ field theory: Cluster expansion and Wightman axioms. Trans. Am. Math. Soc. 234, 1-88 (1977)

11. Dell'Antonio, G. F.: Structure of the algebras of some free systems. Commun. Math. Phys. 9, 81-117 (1968)

12. Doplicher, S., Haag, R., Roberts J.: Fields, observables and gauge transformations, I. Commun. Math. Phys. 13, 1-23 (1969)

13. Doplicher, S., Haag, R., Roberts, J.: Fields, observables and gauge transformations, II. Commun. Math. Phys. 15, 173-200 (1969)

14. Driessler, W.: Duality and absence of locally generated superselection sectors for CCR-type algebras. Commun. Math. Phys. 70, 213-220 (1979)

15. Eckmann, J.-P., Osterwalder, K.: An Application of Tomita's theory of modular Hilbert algebras: duality for free Bose fields. J. Funct. Anal. 13, 1-12 (1973)

16. Foit, G. J.: Ph. D. Thesis, Universität Osnabrück, 1982

17. Glimm, J., Jaffe, A.: Quantum field theory models. Les Houches Lectures 1970. De Witt, C., Stora, R. (eds.) New York: Gordon and Breach 1970 
18. Glimm, J., Jaffe, A.: Boson quantum field models. In: Mathematics in Contemporary Physics, Streater, R. F. (ed.) London, New York: Academic Press 1972

19. Glimm, J., Jaffe, A.: Self-Adjointness of the Yukawa ${ }_{2}$ Hamiltonian. Ann. Phys. 60, 321-383 (1970)

20. Glimm, J., Jaffe, A.: The $\lambda\left(\phi^{4}\right)_{2}$ quantum field theory without cutoffs, III, The physical vacuum. Acta Math. 125, 203-267 (1970)

21. Glimm, J., Jaffe, A.: The Yukawa 2 quantum field theory without cutoffs. J. Funct. Anal. 7, 323-357 (1971)

22. Glimm, J., Jaffe, A., Spencer, T.: The particle structure of the weakly coupled $P(\phi)_{2}$ model and other applications of high temperature expansions. In: Constructive Quantum Field Theory. Velo, G., Wightman, A. (eds.) Lecture Notes in Physics, Vol. 25, Berlin, New York, Heidelberg: Springer 1973

23. Glimm, J., Jaffe, A., Spencer, T.: Convergent expansion about mean field theory, I and II. Ann. Phys. 101, 610-630 and 631-669 (1976)

24. Haag, R., Kastler, D.: An Algebraic approach to quantum field theory. J. Math. Phys. 5, 848-861 (1964)

25. Leyland, P., Roberts, J., Testard, D.: Duality for quantum free fields. Marseille CNRS preprint, CPT 78/p. 1016

26. Magnen, J., Sénéor, R.: The Wightman axioms for the weakly coupled Yukawa model in two dimensions. Commun. Math. Phys. 51, 297-313 (1976)

27. McBryan, O. A., Park, Y. M.: Lorentz covariance of the Yukawa ${ }_{2}$ quantum field theory. J. Math. Phys. 16, 104-110 (1975)

28. Osterwalder, K.: Euclidean Green's functions and Wightman distributions. In: Constructive Quantum Field Theory, Velo, G., Wightman, A. (eds.) Lecture Notes in Physics, Vol. 25, Berlin, Heidelberg, New York: Springer 1973

29. Osterwalder, K.: Duality for free Bose fields. Commun. Math. Phys. 29, 1-14 (1973)

30. Osterwalder, K., Schrader, R.: Euclidean Fermi fields and a Feynman-Kac formula for bosonfermion models. Helv. Phys. Acta. 46, 277-302 (1973)

31. Osterwalder, K., Schrader, R.: Axioms for Euclidean Green's functions, I and II. Commun. Math. Phys. 31, 83-112 (1973) and 42, 281-305 (1975)

32. Powers, R. F., St $\varnothing$ rmer, E.: Free states of the canonical anticommutation relations. Commun. Math. Phys. 16, 1-33 (1970)

33. Roberts, J. E.: The Structure of sectors reached by a field algebra. In: Cargése Lectures in Physics, Vol. 4, Kastler, D. (ed.) New York: Gordon and Breach 1970

34. Roberts, J. E.: Spontaneously broken gauge symmetries and super-selection rules. In: Proceedings of the International School of Mathematical Physics, Univ. of Camerino, 1974, Gallavotti, E. (ed.) Univ. of Camerino, 1976

35. Roberts, J. E.: A Survey of local cohomology. In: Proceedings of the Conference on Mathematical Problems in Theoretical Physics, Rome 1977, (eds.) Doplicher, S. Dell' Antonio, G. F., JonaLasinio, G. (eds.) Lecture in Physics, Vol. 80, Berlin and New York: Springer 1978

36. Roberts, J. E.: Net Cohomology and its applications to field theory. In: Quantum Fields-Algebra, Processes, Streit, L. (ed.) Vienna, New York: Springer 1980

37. Roos, H.: Independence of local algebras in quantum field theory. Commun. Math. Phys. 16, 238$246(1970)$

38. Sakai, S.: C*- and W*-Algebras, Berlin, Heidelberg, New York: Springer 1971

39. Schrader, R.: A Remark on Yukawa plus Boson selfinteraction in two space-tıme dimensions. Commun. Math. Phys. 21, 164-170 (1971)

40. Schrader, R.: Yukawa quantum field theory in two space-time dimensions without cutoffs. Ann. Phys. 70, 412-457 (1972)

41. Segal, I. E., Goodman, R. W.: Anti-locality of certain Lorentz-invariant operators. J. Math. Mech. 14, 629-638 (1965)

42. Seiler, E.: Schwinger functions for the Yukawa model in two dimensions with space-time cutoff. Commun. Math. Phys. 42, 163-182 (1975)

43. Seiler, E., Simon, B.: Nelson's symmetry and all that in the Yukawa 2 and $\left(\phi^{4}\right)_{3}$ field theories. Ann. Phys. 97, 470-518 (1976)

44. Shale, D., Stinespring, W. F.: States on the Clifford algebra. Ann. Math. 80, 365-381 (1964) 
45. Schlieder, S.: Einige Bemerkungen über Projektionsoperatoren. Commun. Math. Phys. 13, 216-225 (1969)

46. Streater, R. F., Wightman, A.: PCT, Spin and Statistics, and All That, New York: Benjamin 1964

47. Kishimoto, A., Takai, H. On the invariant $\Gamma(\alpha)$ in $C^{*}$-dynamical systems. Tôhoku Math. J. 30, 83-94 (1978)

48. Glimm, J., Jaffe, A.: The $\lambda \phi_{2}^{4}$ quantum field theory without cutoffs, IV. J. Math. Phys. 13, 1568-1584 (1972)

49. McBryan, O. A.: Convergence of the vacuum energy density, $\phi$-bounds and existence of Wightman

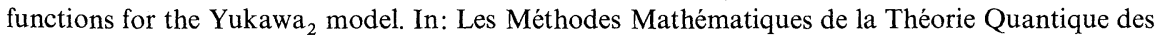
Champs, Proceedings of the 1975 Marseille Conference, Editions du CNRS, 1976

50. Pedersen, G. K.: C*-Algebras and Their Automorphism Groups. London, New York: Academic Press 1979

51. Rideau, G.: On some representations of the anticommutation relations, Commun. Math. Phys. 9, 229-241 (1968)

52. Heifets, E. P., Osipov, E. P.: The energy-momentum spectrum in the Yukawa ${ }_{2}$ quantum field theory. Commun. Math. Phys. 57, 31-50 (1977)

Communicated by $\mathrm{K}$. Osterwalder

Received March 31, 1981; in revised form October 15, 1981 
